\title{
Development and validation of a (semi-)quantitative UHPLC-MS/ MS method for the determination of 191 mycotoxins and other fungal metabolites in almonds, hazelnuts, peanuts and pistachios
}

\author{
Elisabeth Varga • Thomas Glauner • Franz Berthiller • \\ Rudolf Krska • Rainer Schuhmacher • Michael Sulyok
}

Received: 2 January 2013 /Revised: 6 February 2013 /Accepted: 8 February 2013 /Published online: 8 March 2013

(C) The Author(s) 2013. This article is published with open access at Springerlink.com

\begin{abstract}
A multi-target method for the determination of 191 fungal metabolites in almonds, hazelnuts, peanuts and pistachios was developed. The method includes all mycotoxins regulated in the European Union and mycotoxins regularly found in food. After extraction with an acidified acetonitrile water mixture, the raw extract was diluted and injected directly into the UHPLC-MS/MS system. In two chromatographic runs, analysis was performed in positive and in negative ionisation mode. The method was in-house validated for the most important 65 analytes in these four commodities. Apparent recoveries between 80 and $120 \%$ were obtained for about half of the analyte-matrix combinations. Good repeatabilities (standard deviations $<10 \%$ ) were achieved for the vast majority ( $83 \%$ ) of all cases. Only in $6 \%$ of all combinations did the standard deviations exceed $15 \%$. Matrix effects, arising during electrospray ionisation, significantly influenced the determination. For instance, signal suppression was observed for several early-eluting analytes and also signal enhancement up to $295 \%$ for physcion in peanuts was determined. Concerning extraction recovery, $94 \%$ of the analytematrix combinations showed values higher than $50 \%$. Lower
\end{abstract}

Published in the topical collection Metabolomics and Metabolite Profiling with guest editors Rainer Schuhmacher, Rudolf Krska, Roy Goodacre, and Wolfram Weckwerth.

E. Varga $\cdot$ F. Berthiller $(\bowtie) \cdot$ R. Krska $\cdot$ R. Schuhmacher $\cdot$ M. Sulyok

Christian Doppler Laboratory for Mycotoxin Metabolism and Center for Analytical Chemistry, Department for

Agrobiotechnology (IFA-Tulln), University of Natural Resources and Life Sciences, Vienna (BOKU), Konrad Lorenz Str. 20, 3430 Tulln, Austria

e-mail: franz.berthiller@boku.ac.at

T. Glauner

Chemical Analysis Group, Agilent Technologies Sales \& Services GmbH \& Co. KG, Hewlett-Packard-Str. 8,

76337 Waldbronn, Germany limits of quantification ranged between $0.04 \mu \mathrm{g} \mathrm{kg}^{-1}$ for enniatin $\mathrm{B} 3$ in peanuts and $500 \mathrm{\mu gkg}^{-1}$ for $\mathrm{HC}$ toxin in hazelnuts. Additionally, the applicability of the developed method was demonstrated through the analysis of 53 naturally contaminated nut samples from Austria and Turkey. Overall, 40 toxins were quantified; the most frequently found mycotoxins were beauvericin (79\%), enniatin B (62\%) and macrosporin $(57 \%)$. In the most contaminated hazelnut sample, 26 different fungal metabolites were detected.

Keywords Multi-target analysis $\cdot$ Tandem mass spectrometry $\cdot$ Ultra-high-performance liquid chromatography $\cdot$ Nuts

\section{Introduction}

Mycotoxins are secondary fungal metabolites which are found in a broad range of food and feed, such as cereals, spices, coffee, nuts or dried fruits [1]. They have the capability of causing acute toxic, carcinogenic, mutagenic, teratogenic, immunotoxic or oestrogenic effects in animals and humans [2]. Mycotoxins show a huge structural diversity resulting in a variety of chemical and physicochemical properties. The most important mycotoxins in food and feed, which are regulated in the European Union [3], are aflatoxins $\left(\mathrm{AFB}_{1}, \mathrm{AFB}_{2}, \mathrm{AFG}_{1}\right.$, $\left.\mathrm{AFG}_{2}\right)$; ochratoxin $\mathrm{A}(\mathrm{OTA})$; type $\mathrm{A}$ and $\mathrm{B}$ trichothecenes (e.g. HT-2 toxin, T-2 toxin and deoxynivalenol (DON)); fumonisins; and zearalenone.

During the last years, single analyte methods for the detection and quantification of mycotoxins are more and more replaced by multi-target methods for the simultaneous determination of different yet co-occurring classes of mycotoxins. The majority of these methods are based on the combination of high- or ultra-high-performance liquid chromatography with tandem (e.g. [4-7]) or high-resolution [8] mass spectrometry. 
The importance of mass spectrometry for the confirmation of mycotoxin identity is emphasised, e.g. in [1]. A major advantage of LC-MS-based multi-target methods is the increased sample throughput compared to single-analyte methods. Still, such multi-target methods have to cope with huge differences in the relevant toxin concentrations. Naturally, the choice of an appropriate extraction solvent for a large number of analytes with different physicochemical properties is also a great challenge. Mixtures of water with high amounts of methanol or acetonitrile $(>75 \%)$ are appropriate extraction solvents for most mycotoxins. However, for fumonisins, higher extraction recoveries are achieved when the water proportion is increased and/or the pH of the solvent is decreased [4]. Mol et al. [9] concluded that aqueous acetone is the favourite extraction solvent with respect to extraction recovery, but aqueous acetonitrile should be preferred considering matrix effects. Extractions using aqueous methanol resulted in extracts exhibiting both lower extraction recoveries and causing more severe matrix effects [9]. Anyway, the chosen solvent has to be a compromise to reach agreeable extraction recoveries for the majority of the analytes.

Regarding cleanup, multi-target methods use simple "dilute-and-shoot" approaches $[4,5,7,10]$, solid phase extraction $[6,11,12]$, MycoSep ${ }^{\circledR}$ columns [13], immunoaffinity columns [14] and, recently, also QuEChERS $[8,15,16]$. A drawback of most multi-target methods is that they require extensive validation which is time- and cost-consuming and, hence, often reduced to a minimum. Therefore, these methods are often used just for semiquantitative screening purposes. Most multi-target methods were developed for the measurement of raw cereals, whereas data on the performance characteristics in other matrices like nuts are scarce.

Nuts are hard-shelled fruits of some plants having an indehiscent seed and are an important source of nutrients for humans and animals. Almonds, hazelnuts and pistachios belong to the most popular tree nuts [17]. More correctly, almonds are not nuts, but drupes which consist of a hull and a hard shell containing the seed. Peanuts or groundnuts are actually legumes, but in the general linguistic usage, they are referred to as nuts. For simplification, the term "nut" will be used in this paper for all four matrices under investigation (almonds, hazelnuts, peanuts and pistachios).

Fungi of the genera Fusarium, Alternaria and Cladosporium dominate the mycobiota of nuts in the field, whilst in storage, Aspergillus, Penicillium and Trichoderma are predominant [18]. Almost all Aspergillus parasiticus strains tested in tree nuts produced aflatoxins, whilst all of the detected Aspergillus alliaceus strains produced OTA [19]. In general, nuts with thick shells (e.g. macadamia nuts) are better protected against the intrusion of moulds. Other nuts, like pistachios, are more prone to mould infestation due to shell splitting at the end of maturation [20]. Sorting and elimination of split nuts can decrease the contamination of mycotoxins in the lot significantly [21].
Information about mycotoxin contamination of nuts other than aflatoxins (recently reviewed in [22]) and OTA is limited. In most cases, only single (OTA) [23] or group-target (aflatoxins) [24] methods using chromatographic (TLC, LC-FLD or LCMS/MS) or immuno-based methods (ELISA, fluorescence immunoassays) were applied for the investigation of the content of those mycotoxins. In 2008, Spanjer et al. [5] published the validation of an LC-MS/MS-based multi-target method for the determination of mycotoxins in various matrices, including peanuts (13 mycotoxins) and pistachios (24 mycotoxins). A first application for a semiquantitative screening of multiple mycotoxins in various food (including six nut samples) was published in 2010 [25]. Other studies investigated the occurrence of mycotoxins in peanut cake [26, 27] and peanuts [28] from Africa.

Based on previous HPLC-MS/MS methods for the multiple determination of mycotoxins in cereals developed in our group $[4,7]$, we created a new multi-target ultra-highperformance liquid chromatographic tandem mass spectrometric (UHPLC-MS/MS) method which covers a total number of 191 fungal metabolites. Validation parameters were obtained for those analytes which seem to be of importance in almonds, hazelnuts, peanuts and pistachios. Finally, we show the applicability of the method by analysing nuts bought on Austrian and Turkish markets.

\section{Experimental}

Chemicals, standards and samples

Methanol and acetonitrile (both LC gradient grade), as well as acetic acid (p.a.), were purchased from VWR International (Vienna, Austria), whereas ammonium acetate (MS grade) was obtained from Sigma-Aldrich (Vienna, Austria). Water was purified by reverse osmosis followed by a Milli-Q Plus system from Millipore (Molsheim, France).

Solid standards or stock solutions were collected from different sources over the years: They were either isolated by our own group, provided by other research groups or purchased from one of the following commercial companies: Alexis Austria (Vienna, Austria), Alfarma (Prague, Czech Republic), Axxora Europe (Lausanne, Switzerland), Bioaustralis (distributed by Tebu-Bio, Germany), Iris Biotech GmbH (Marktredwitz, Germany), LGC Promochem GmbH (Wesel, Germany), Romer Labs (Tulln, Austria) and SigmaAldrich. Individual stock solutions of the standards were prepared by dissolving the weighted solid substance in acetonitrile, or, if insoluble, either in methanol, acetonitrile/water $(50: 50, v / v)$, methanol/water $(50: 50, v / v)$ or pure water. The stock solutions were combined to 30 working solutions containing up to 13 individual mycotoxins and were stored at $-20{ }^{\circ} \mathrm{C}$. Before usage, the working solutions were brought 
to room temperature in the dark, thoroughly mixed and a multi-analyte stock solution was freshly prepared thereof. Neat standard solutions covering a concentration range of three orders of magnitude were obtained by dilution of the multi-analyte stock solution with dilution solvent (acetonitrile/water/acetic acid, 20:79:1, v/v/v) resulting in relative concentrations of 1:3.33:10:33.3:100:333:1,000.

Nut samples were purchased from various stores in Tulln and Vienna (both Austria) or were kindly provided by Dr. Ahmet D. Duman from the Department of Food Engineering of the Faculty of Agriculture of Kahramanmaras Sutcu Imam University (KSU), Turkey. Samples obtained in Austria were stored at $-20{ }^{\circ} \mathrm{C}$ on the same or following day until usage. Turkish samples were collected on markets, orchards and in warehouses from the Black Sea Region (hazelnuts) or from the Osmaniye province (peanuts) in the harvest season of 2007. Turkish samples were frozen at $-20^{\circ} \mathrm{C}$, sent cooled to the IFA-Tulln on the day of purchase and stored until measurement at $-20{ }^{\circ} \mathrm{C}$.

\section{Sample preparation}

Sample preparation was based on the method for cereals described by Sulyok et al. [4]. In-shell pistachios were pealed and nut samples were ground using an Osterizer ${ }^{\circledR}$ Blender (Sunbeam Oster Household Products, USA). Of the ground samples, $5.00 \pm 0.01 \mathrm{~g}$ was weighted in $50-\mathrm{mL}$ polypropylene tubes (Sarstedt, Wr. Neudorf, Austria). For extraction, $20 \mathrm{~mL}$ extraction solvent (acetonitrile/water/acetic acid, 79:20:1, v/v/v) was added and the samples were extracted in a vertical position on a GFL3017 rotary shaker (Burgwedel, Germany) for $90 \mathrm{~min}$ at room temperature (200 rpm). After extraction, the solid residue was allowed to settle for a few minutes. An aliquot of the raw extract was transferred to an HPLC vial and diluted with the same volume of an acetonitrile/water/acetic acid mixture $(20: 79: 1, v / v / v)$, resulting in a total dilution factor of 8 . Five microlitres of this solution was injected into the UHPLCMS/MS system without any further cleanup.

\section{UHPLC-MS/MS parameters}

For the analysis, a 1290 Infinity ultra-high-performance liquid chromatography (UHPLC) system coupled to a 6460 Triple Quadrupole mass spectrometer (both Agilent Technologies, Waldbronn, Germany) was used. Chromatographic separation was performed at $25^{\circ} \mathrm{C}$ and a flow rate of $250 \mu \mathrm{Lmin}^{-1}$ using a ZORBAX Eclipse Plus C18 Rapid Resolution High Definition $(150 \times 2.1 \mathrm{~mm}, 1.8 \mu \mathrm{m})$ column from Agilent Technologies. Eluent composition was chosen according to Sulyok et al. [4], and the gradient was modified to enhance the separation of the analytes. Hence, the eluents were composed of methanol/water/acetic acid (eluent $\mathrm{A}-10: 89: 1, v / v / v$; eluent
B-97:2:1, v/v/v) containing $5 \mathrm{mM}$ ammonium acetate. The total run time of one chromatographic run was 21 min: after an initial hold time of 2 min at $100 \% \mathrm{~A}, 50 \% \mathrm{~B}$ was reached within $3 \mathrm{~min}$ and $100 \% \mathrm{~B}$ within the next $9 \mathrm{~min}$. A hold time of $4.5 \mathrm{~min}$ at $100 \% \mathrm{~B}$ was followed by $2.5 \mathrm{~min}$ at $100 \% \mathrm{~A}$ for column re-equilibration. Before injecting the sample into the UHPLC system, the needle was washed in the flush port with acetonitrile/water $(50: 50, v / v)$ for $5 \mathrm{~s}$.

Precursor and product ion selection and the optimisation of fragmentor voltages and collision energies were performed with flow injection of single-analyte solutions using MassHunter Optimizer Triple Quad B04.01. Optimisation was either done in one or in both ionisation modes depending on our experiences with previous methods. MassHunter Data Acquisition software version B04.01 was used to control the LC-MS/MS instrument. Analysis was carried out using electrospray ionisation (ESI) and the dynamic multiple reaction monitoring (DMRM) acquisition. DMRM allows the measurement of selected reaction monitoring transitions for a specified time period (expected retention time \pm variable window width), hence resulting in maximized dwell times for each transition. Due to the amount of analytes and to ensure optimum ionisation yields, analysis was performed in two chromatographic runs-one for each ionisation mode. For each compound, two mass transitions were monitored (except for moniliformin and 3nitropropionic acid), resulting in 4.0 identification points, which is in agreement with Commission Decision 2002/657/EC [29]. The general source settings were as follows: gas temperature, $200{ }^{\circ} \mathrm{C}$; gas flow, $8 \mathrm{Lmin}^{-1}$; nebulizer, 40 psi $(275.8 \mathrm{kPa})$; sheath gas temperature, $350{ }^{\circ} \mathrm{C}$; sheath gas flow, $11 \mathrm{Lmin}^{-1}$; capillary voltage, $3,500 \mathrm{~V}$; and nozzle voltage, $500 \mathrm{~V}$ (positive) or $0 \mathrm{~V}$ (negative). Both scanning quadrupoles (MS1 and MS2) were set to unit resolution. Cycle time was set to $750 \mathrm{~ms}$.

\section{Method validation}

For validation purposes, one blank sample of each commodity (almond, hazelnut, peanut and pistachio) was selected. For each matrix, four times $0.50 \pm 0.01 \mathrm{~g}$ thoroughly homogenised and ground sample was weighed in $16-\mathrm{mL}$ glass vials. Three of those samples were spiked on one medium concentration level by adding an aliquot of the multi-analyte stock solution prior to extraction. The samples were stored overnight at $40{ }^{\circ} \mathrm{C}$ in a Kelvirton ${ }^{\circledR}$ T60120 drying chamber (Heraeus Instruments, Hanau, Germany) to allow evaporation of the solvent. Thereafter, those three spiked samples as well as the fourth blank sample were processed according to the procedure described in "Sample preparation". The remaining raw extract of the blank sample was used for the preparation of spiked raw extracts on seven levels without replicates and with relative concentrations of 
1:3.33:10:33.3:100:333:1,000. This approach (adding the multi-analyte stock solution before and after extraction) allowed the direct determination of the apparent recovery $\left(R_{\mathrm{A}}\right)$ as well as the assessment of matrix effects caused by signal suppression or enhancement (SSE) of the analyte signal.

\section{Data evaluation}

For each analyte, linear, $1 / x$ weighted calibration curves were calculated by plotting the peak area of the signal in neat standard solution versus the analyte concentration using MassHunter Quantitative Analysis. Apparent recoveries were calculated from the three samples spiked before extraction in the following way: In a first step, the ratio of the measured to spiked concentration for each of the three individual measurements was calculated and multiplied with 100 . The average and standard deviation (SD) of those three values was the obtained $R_{\mathrm{A}}$ and SD for the respective analyte-matrix combination. Matrix effects (SSE) were determined in a similar way as the apparent recoveries, but in this case, the results from the raw extracts of the blank sample spiked on seven different concentration levels after extraction were used for the calculation. Hence, the ratio of the measured to the spiked concentration for these seven levels were calculated and multiplied with 100 . For the calculation of the average SSE value, only those levels for which both the qualifier and the quantifier showed a distinct peak were used. The recovery of extraction was calculated by dividing the $R_{\mathrm{A}}$ value by the SSE value and a multiplication factor of 100 . The lower limit of quantification (LLOQ) was calculated as follows: First, the concentration level of the raw extract spiked after extraction for which both mass transitions showed an $\mathrm{S} / \mathrm{N}$ ratio above 10:1 was determined. This value was then multiplied with the dilution factor of 8 and corrected for the recovery of the extraction step of the respective matrix.

For positive results, the following criteria had to be fulfilled: First, the retention time had to be within $\pm 2.5 \%$ compared to the analyte in neat standard solution and both, the qualifier and the quantifier, transition had to be above an $\mathrm{S} / \mathrm{N}$ ratio of 10:1. Furthermore, the ion ratio of the quantifier and the qualifier transition had to be within a defined target range according to Commission Decision 2002/657/EC [29]. MassHunter Quantitative Analysis allows setting the criteria for retention time deviation as well as the ratio of quantifier and qualifier; non-compliance is marked in blue (lower) or red (higher) colour. All results obtained for the naturally contaminated samples were multiplied with the dilution factor of 8 and corrected with the apparent recoveries determined during the validation. For the calculation of the average values, all contaminated samples were taken into considerations; for values below the LLOQ, half of the LLOQ of the respective matrix was used.

\section{Results and discussion}

Development of the analytical method

Based on an HPLC-MS/MS multi-target method developed previously by our group [4, 7], a new UHPLC-MS/MS method was developed on an Agilent 1290 Infinity UHPLC system coupled to a 6460 Triple Quadrupole mass spectrometer. On purpose, we did not speed up the previous method, but used the employed stationary phase with sub-2- $\mu \mathrm{m}$ particles for better resolution of analytes from the matrix. The gradient was adapted from the original method and flattened to allow better separation of the analytes. Another major advantage of this method proved to be the use of the DMRM mode. Early multi-target methods for the determination of mycotoxins in food dealing with a large number of analytes (e.g. [7]) employed time periods for which only certain analytes are measured to allow enough dwell time for each analyte. These periods are prone to retention time shifts due to various reasons, e.g. caused by slightly different solvent compositions, by declining column performances or slight temperature shifts. Using DMRM, the size of the retention time window for each mass transition can be set individually. As chromatographic conditions were very repeatable during method development, we generally set the time window to $1 \mathrm{~min}$. Moreover, for broad chromatographic peaks (e.g. HC toxin and ustiloxin B), window widths of 2 min were used. If two analytes share the same mass transitions, window widths which includes the different retention times can be selected (e.g. 1.5 min for cytochalasin C and D or enniatin B2 and $\mathrm{K} 1)$. Using these variable window widths, we obtained minimum dwell times of at least $10 \mathrm{~ms}$, even in the most crowded sections of the chromatogram. In order to gain enough points over a given chromatographic peak, we set the duty cycle of the mass spectrometer to $750 \mathrm{~ms}$. The usual peak showed a base width of $0.2 \mathrm{~min}$, resulting in 16 data points, which were well suited for reliable integration of the peak area. Moreover, also cyclopiazonic acid, which is known to give broad peaks under acidic RP-HPLC conditions, showed an acceptable peak width of about $0.5 \mathrm{~min}$ in our case.

To ensure optimum sensitivity, we decided to apply two chromatographic runs per sample - one for each ionisation mode. For each analyte, the mode resulting in the most abundant signal was used and two mass transitions were selected: one for the use as quantifier and one as qualifier. The formation of sodium adducts of certain analytes led to insufficient fragment intensity because the positive charge remains on the sodium ion after collision-induced dissociation. Therefore, ammonium acetate was added to both of the solvents. Most analytes were detected as single-charged ions either as protonated $[\mathrm{M}+\mathrm{H}]^{+}$or ammonium adducts $[\mathrm{M}+$ $\left.\mathrm{NH}_{4}\right]^{+}$(e.g. 4,15-diacetoxyscirpenol, enniatins and 
mycophenolic acid) in positive mode or as deprotonated [M$\mathrm{H}]^{-}$or acetate adducts $[\mathrm{M}+\mathrm{OAc}]^{-}$(e.g. the B-trichothecenes DON and nivalenol) in negative mode. Exceptions were cyclosporin A, C, D and $\mathrm{H}$ which were measured as doubly charged $[\mathrm{M}+2 \mathrm{H}]^{2+}$ ions. For alamethicin F30 (cleavage of the peptide bond) [30] and also for fusarielin A (loss of water) in-source fragments were used as precursors as these ions were predominantly formed using ESI.

Table 1 summarises the obtained retention times, precursor and product ions including the optimised ESI-MS/MS settings for all analytes ionising in negative electrospray mode. The same parameters are given in Table 2 for the analytes showing higher ionisation abundance in positive electrospray mode. Only one fragment ion of sufficient sensitivity was obtained for the very small molecules moniliformin $\left(98 \mathrm{gmol}^{-1}\right)$ and 3nitropropionic acid (119 $\left.\mathrm{gmol}^{-1}\right)$. In general, the most abundant mass transition was used as the quantifier, but when the $\mathrm{S} / \mathrm{N}$ ratio was significantly worse for this mass transition, another one was used. Using MassHunter Quantitative Analysis, the qualifier-to-quantifier ratios based on the peak area

Table 1 List of analytes determined in the negative ionisation mode and optimised ESI-MS/MS parameters

\begin{tabular}{|c|c|c|c|c|}
\hline Analyte name & Retention time (min) & $\begin{array}{l}m / z \text { precursor ion (framentor } \\
\text { voltage) }\end{array}$ & Ion species & $\begin{array}{l}m / z \text { product ion (collision } \\
\text { energy }(\mathrm{V}) \text { ) }\end{array}$ \\
\hline 3-Acetyldeoxynivalenol & 7.4 & $397(95)$ & {$[\mathrm{M}+\mathrm{OAc}]^{-}$} & $307(8) / 59(20)$ \\
\hline alpha-Zearalenol & 11.8 & $319(195)$ & {$[\mathrm{M}-\mathrm{H}]^{-}$} & $160(28) / 130(32)$ \\
\hline alpha-Zearalenol-14-glucoside & 9.2 & $541(100)$ & {$[\mathrm{M}+\mathrm{OAc}]^{-}$} & $319(16) / 481(0)$ \\
\hline Alternariol & 10.4 & $257(190)$ & {$[\mathrm{M}-\mathrm{H}]^{-}$} & $213(16) / 215(20)$ \\
\hline Alternariolmethylether & 12.5 & $271(170)$ & {$[\mathrm{M}-\mathrm{H}]^{-}$} & $256(16) / 227(32)$ \\
\hline Altersolanol C & 7.8 & $319(105)$ & {$[\mathrm{M}-\mathrm{H}]^{-}$} & $283(12) / 301(8)$ \\
\hline Altertoxin I & 10.0 & $351(140)$ & {$[\mathrm{M}-\mathrm{H}]^{-}$} & $315(8) / 263(32)$ \\
\hline Apicidin & 13.0 & $622(220)$ & {$[\mathrm{M}-\mathrm{H}]^{-}$} & $462(16) / 252(32)$ \\
\hline Atpenin A5 & 13.5 & $364(120)$ & {$[\mathrm{M}-\mathrm{H}]^{-}$} & $292(4) / 328(0)$ \\
\hline beta-Zearalenol & 10.7 & $319(185)$ & {$[\mathrm{M}-\mathrm{H}]^{-}$} & $160(28) / 130(32)$ \\
\hline beta-Zearalenol-14-glucoside & 8.1 & $541(115)$ & {$[\mathrm{M}+\mathrm{OAc}]^{-}$} & $319(16) / 481(0)$ \\
\hline Cycloechinulin & 9.3 & $350(170)$ & {$[\mathrm{M}-\mathrm{H}]^{-}$} & $335(20) / 320(28)$ \\
\hline Cyclopiazonic acid & 12.2 & $335(270)$ & {$[\mathrm{M}-\mathrm{H}]^{-}$} & $140(28) / 154(32)$ \\
\hline Deoxynivalenol & 5.9 & $355(95)$ & {$[\mathrm{M}+\mathrm{OAc}]^{-}$} & $265(8) / 59(16)$ \\
\hline Deoxynivalenol-3-glucoside & 5.8 & $517(145)$ & {$[\mathrm{M}+\mathrm{OAc}]^{-}$} & $427(16) / 457(8)$ \\
\hline Emodin & 14.1 & $269(185)$ & {$[\mathrm{M}-\mathrm{H}]^{-}$} & $225(20) / 241(20)$ \\
\hline Equisetin & 15.2 & $372(205)$ & {$[\mathrm{M}-\mathrm{H}]^{-}$} & $342(20) / 124(48)$ \\
\hline Fusarenon-X & 6.6 & $413(110)$ & {$[\mathrm{M}+\mathrm{OAc}]^{-}$} & $263(8) / 59(28)$ \\
\hline Fusidic acid & 15.1 & $515(190)$ & {$[\mathrm{M}-\mathrm{H}]^{-}$} & $221(24) / 455(16)$ \\
\hline Macrosporin & 13.6 & $283(170)$ & {$[\mathrm{M}-\mathrm{H}]^{-}$} & $268(16) / 225(36)$ \\
\hline Moniliformin & 1.5 & $97(65)$ & {$[\mathrm{M}-\mathrm{H}]^{-}$} & $41(8)$ \\
\hline Nidulin & 15.4 & $441(185)$ & {$[\mathrm{M}-\mathrm{H}]^{-}$} & $382(20) / 390(24)$ \\
\hline 3-Nitropropionic acid & 2.2 & $118(65)$ & {$[\mathrm{M}-\mathrm{H}]^{-}$} & $46(8)$ \\
\hline Nivalenol & 4.8 & $371(110)$ & {$[\mathrm{M}+\mathrm{OAc}]^{-}$} & $281(8) / 59(20)$ \\
\hline Nornidulin & 14.1 & $427(160)$ & {$[\mathrm{M}-\mathrm{H}]^{-}$} & $347(16) / 376(20)$ \\
\hline Patulin & 4.5 & $153(70)$ & {$[\mathrm{M}-\mathrm{H}]^{-}$} & $109(4) / 81(4)$ \\
\hline Penigequinolone A & 13.0 & $466(200)$ & {$[\mathrm{M}-\mathrm{H}]^{-}$} & $394(32) / 378(48)$ \\
\hline Physcion & 15.4 & $283(145)$ & {$[\mathrm{M}-\mathrm{H}]^{-}$} & $240(20) / 211.6(40)$ \\
\hline Pseurotin A & 8.6 & $430(100)$ & {$[\mathrm{M}-\mathrm{H}]^{-}$} & $270(4) / 308(0)$ \\
\hline Radicicol & 9.0 & $363(165)$ & {$[\mathrm{M}-\mathrm{H}]^{-}$} & $183(20) / 224(16)$ \\
\hline Rubellin D & 12.7 & $541(165)$ & {$[\mathrm{M}-\mathrm{H}]^{-}$} & $360(24) / 378(16)$ \\
\hline Tentoxin & 10.4 & $413(160)$ & {$[\mathrm{M}-\mathrm{H}]^{-}$} & $271(12) / 141(12)$ \\
\hline Tenuazonic acid & 8.4 & $196(150)$ & {$[\mathrm{M}-\mathrm{H}]^{-}$} & $112(20) / 139(16)$ \\
\hline Zearalenone & 11.9 & $317(195)$ & {$[\mathrm{M}-\mathrm{H}]^{-}$} & $131(28) / 175(20)$ \\
\hline Zearalenone-14-glucoside & 9.4 & $479(190)$ & {$[\mathrm{M}-\mathrm{H}]^{-}$} & $317(8) / 175(44)$ \\
\hline Zearalenone-14-sulphate & 8.8 & $397(160)$ & {$[\mathrm{M}-\mathrm{H}]^{-}$} & $317(20) / 131(44)$ \\
\hline
\end{tabular}


Table 2 List of analytes determined in the positive ionisation mode and optimised ESI-MS/MS parameters

\begin{tabular}{|c|c|c|c|c|}
\hline Analyte name & Retention time (min) & $\begin{array}{l}m / z \text { precursor ion } \\
\text { (framentor voltage) }\end{array}$ & Ion species & $\begin{array}{l}m / z \text { product ion (collision } \\
\text { energy }(V) \text { ) }\end{array}$ \\
\hline AAL toxin TA 1 & 9.0 & $522(165)$ & {$[\mathrm{M}+\mathrm{H}]^{+}$} & $328.5(24) / 292.4(28)$ \\
\hline 15-Acetyldeoxynivalenol & 7.4 & $339(110)$ & {$[\mathrm{M}+\mathrm{H}]^{+}$} & $321(4) / 137(4)$ \\
\hline Aflatoxin $\mathrm{B}_{1}$ & 8.7 & $313(165)$ & {$[\mathrm{M}+\mathrm{H}]^{+}$} & $285.2(20) / 128.1(70)$ \\
\hline Aflatoxin $\mathrm{B}_{2}$ & 8.4 & $315(190)$ & {$[\mathrm{M}+\mathrm{H}]^{+}$} & $287(24) / 259(28)$ \\
\hline Aflatoxin $\mathrm{G}_{1}$ & 8.0 & $329(175)$ & {$[\mathrm{M}+\mathrm{H}]^{+}$} & $200(44) / 243.2(24)$ \\
\hline Aflatoxin $\mathrm{G}_{2}$ & 7.7 & $331(190)$ & {$[\mathrm{M}+\mathrm{H}]^{+}$} & $313(24) / 245(28)$ \\
\hline Aflatoxin $\mathrm{M}_{1}$ & 7.7 & $329(180)$ & {$[\mathrm{M}+\mathrm{H}]^{+}$} & $273(24) / 229(28)$ \\
\hline Aflatoxin $\mathrm{M}_{2}$ & 7.3 & $331(145)$ & {$[\mathrm{M}+\mathrm{H}]^{+}$} & $273(20) / 285(20)$ \\
\hline Agroclavine & 6.9 & $239(130)$ & {$[\mathrm{M}+\mathrm{H}]^{+}$} & $183(16) / 208(16)$ \\
\hline Alamethicin F30 & 14.9 & $775(225)$ & {$\left[\mathrm{y} 7^{\mathrm{a}}+\mathrm{H}\right]^{+}$} & $282(44) / 197(52)$ \\
\hline Altenuene & 8.6 & $293(100)$ & {$[\mathrm{M}+\mathrm{H}]^{+}$} & $257(8) / 275(4)$ \\
\hline Altenusin & 8.8 & $291(90)$ & {$[\mathrm{M}+\mathrm{H}]^{+}$} & $128(56) / 199(32)$ \\
\hline 2-Amino-14,16-dimethyloctadecan-3-ol & 15.5 & $314(115)$ & {$[\mathrm{M}+\mathrm{H}]^{+}$} & $296.5(16) / 125(12)$ \\
\hline Aspercolorin & 10.9 & $465(155)$ & {$[\mathrm{M}+\mathrm{H}]^{+}$} & $247(16) / 120(48)$ \\
\hline Aspergillimide & 6.8 & $360(230)$ & {$[\mathrm{M}+\mathrm{H}]^{+}$} & $301(36) / 332(12)$ \\
\hline Asperlactone & 5.6 & $185(65)$ & {$[\mathrm{M}+\mathrm{H}]^{+}$} & $141(0) / 113(4)$ \\
\hline Asperloxin A & 10.1 & $394(205)$ & {$[\mathrm{M}+\mathrm{H}]^{+}$} & $123(20) / 95(44)$ \\
\hline Aspinonene & 4.9 & $206(80)$ & {$\left[\mathrm{M}+\mathrm{NH}_{4}\right]^{+}$} & $127(0) / 81(12)$ \\
\hline Aspyrone & 6.2 & $185(75)$ & {$[\mathrm{M}+\mathrm{H}]^{+}$} & $125(4) / 139(0)$ \\
\hline Asterric acid & 9.5 & $349(80)$ & {$[\mathrm{M}+\mathrm{H}]^{+}$} & $299(8) / 287(12)$ \\
\hline Aureobasidin A & 15.8 & $1102(270)$ & {$[\mathrm{M}+\mathrm{H}]^{+}$} & $665.5(28) / 210(48)$ \\
\hline Austdiol & 6.5 & $237(125)$ & {$[\mathrm{M}+\mathrm{H}]^{+}$} & $117(28) / 159(20)$ \\
\hline Austocystin A & 13.1 & $373(165)$ & {$[\mathrm{M}+\mathrm{H}]^{+}$} & $329(28) / 312(28)$ \\
\hline Avenacein Y & 10.0 & $319(120)$ & {$[\mathrm{M}+\mathrm{H}]^{+}$} & $175(36) / 287(16)$ \\
\hline Beauvericin & 15.0 & $801.5(180)$ & {$\left[\mathrm{M}+\mathrm{NH}_{4}\right]^{+}$} & $244(36) / 262(32)$ \\
\hline Brefeldin A & 10.2 & $281(75)$ & {$[\mathrm{M}+\mathrm{H}]^{+}$} & $245(0) / 199(4)$ \\
\hline Brevicompanine B & 12.6 & $368(120)$ & {$[\mathrm{M}+\mathrm{H}]^{+}$} & $130(24) / 300(8)$ \\
\hline Calphostin C & 13.9 & $791(220)$ & {$[\mathrm{M}+\mathrm{H}]^{+}$} & $485(32) / 515(20)$ \\
\hline Cephalosporin C & 4.0 & $416(143)$ & {$[\mathrm{M}+\mathrm{H}]^{+}$} & $185(12) / 143(12)$ \\
\hline Cerulenin & 9.7 & $224(85)$ & {$[\mathrm{M}+\mathrm{H}]^{+}$} & $196(0) / 179(0)$ \\
\hline Chaetocin & 11.5 & $697(135)$ & {$[\mathrm{M}+\mathrm{H}]^{+}$} & $348(12) / 350(12)$ \\
\hline Chaetoglobosin A & 12.3 & $529(135)$ & {$[\mathrm{M}+\mathrm{H}]^{+}$} & $130(44) / 511(4)$ \\
\hline Chaetomin & 12.2 & $711(155)$ & {$[\mathrm{M}+\mathrm{H}]^{+}$} & $298(12) / 348(8)$ \\
\hline Chanoclavine & 6.3 & $257(105)$ & {$[\mathrm{M}+\mathrm{H}]^{+}$} & $168(16) / 226(8)$ \\
\hline Chlamydosporol & 7.1 & $227(110)$ & {$[\mathrm{M}+\mathrm{H}]^{+}$} & $167(12) / 106(32)$ \\
\hline Citreoviridin A & 11.9 & $420(95)$ & {$\left[\mathrm{M}+\mathrm{NH}_{4}\right]^{+}$} & $315.4(0) / 285(8)$ \\
\hline Citrinin & 8.9 & $251(120)$ & {$[\mathrm{M}+\mathrm{H}]^{+}$} & $233(12) / 205(28)$ \\
\hline Citromycetin & 8.7 & $291(115)$ & {$[\mathrm{M}+\mathrm{H}]^{+}$} & $245(24) / 217(32)$ \\
\hline Cochliodinol & 16.0 & $507(150)$ & {$[\mathrm{M}+\mathrm{H}]^{+}$} & $371(20) / 439(8)$ \\
\hline Curvularin & 9.7 & $310(75)$ & {$\left[\mathrm{M}+\mathrm{NH}_{4}\right]^{+}$} & $125(12) / 169(8)$ \\
\hline Cyclopenin & 8.5 & $295(105)$ & {$[\mathrm{M}+\mathrm{H}]^{+}$} & $146(20) / 177(8)$ \\
\hline Cyclopeptine & 9.4 & $281(140)$ & {$[\mathrm{M}+\mathrm{H}]^{+}$} & $120(20) / 134(20)$ \\
\hline Cyclosporin $\mathrm{A}+\mathrm{H}^{\mathrm{b}}$ & 16.0 & $602(140)$ & {$[\mathrm{M}+2 \mathrm{H}]^{2+}$} & $100(56) / 156(32)$ \\
\hline Cyclosporin C & 15.4 & $610(125)$ & {$[\mathrm{M}+2 \mathrm{H}]^{2+}$} & $100(52) / 156(32)$ \\
\hline Cyclosporin D & 16.3 & $609(140)$ & {$[\mathrm{M}+2 \mathrm{H}]^{2+}$} & $100(52) / 156(32)$ \\
\hline Cytochalasin A & 12.7 & $478(190)$ & {$[\mathrm{M}+\mathrm{H}]^{+}$} & $91(60) / 120(24)$ \\
\hline Cytochalasin B & 10.5 & $480(160)$ & {$[\mathrm{M}+\mathrm{H}]^{+}$} & $462.5(12) / 444.5(12)$ \\
\hline Cytochalasin $\mathrm{C}$ & 11.2 & $525(110)$ & {$\left[\mathrm{M}+\mathrm{NH}_{4}\right]^{+}$} & $430.5(12) / 490.5(4)$ \\
\hline
\end{tabular}


Table 2 (continued)

\begin{tabular}{|c|c|c|c|c|}
\hline Analyte name & Retention time (min) & $\begin{array}{l}m / z \text { precursor ion } \\
\text { (framentor voltage) }\end{array}$ & Ion species & $\begin{array}{l}m / z \text { product ion (collision } \\
\text { energy }(\mathrm{V}) \text { ) }\end{array}$ \\
\hline Cytochalasin D & 10.4 & $525(110)$ & {$\left[\mathrm{M}+\mathrm{NH}_{4}\right]^{+}$} & $430.5(12) / 490.5(4)$ \\
\hline Cytochalasin E & 11.6 & $513(110)$ & {$\left[\mathrm{M}+\mathrm{NH}_{4}\right]^{+}$} & $434.5(4) / 416(4)$ \\
\hline Cytochalasin $\mathrm{H}$ & 10.6 & $494(135)$ & {$[\mathrm{M}+\mathrm{H}]^{+}$} & $434.5(4) / 416.5(8)$ \\
\hline Cytochalasin $\mathrm{J}$ & 9.8 & $452(145)$ & {$[\mathrm{M}+\mathrm{H}]^{+}$} & $434.5(4) / 416.5(12)$ \\
\hline Decarestrictine D & 5.5 & $217(80)$ & {$[\mathrm{M}+\mathrm{H}]^{+}$} & $121(8) / 163(4)$ \\
\hline Dechlorogriseofulvin & 9.1 & $319(135)$ & {$[\mathrm{M}+\mathrm{H}]^{+}$} & $181(12) / 251(16)$ \\
\hline Deoxybrevianamid E & 10.1 & $352(110)$ & {$[\mathrm{M}+\mathrm{H}]^{+}$} & $130(28) / 284(8)$ \\
\hline 4,15-Diacetoxyscirpenol & 8.7 & $384(110)$ & {$\left[\mathrm{M}+\mathrm{NH}_{4}\right]^{+}$} & $307(4) / 247(8)$ \\
\hline Dihydroergine & 5.5 & $270(150)$ & {$[\mathrm{M}+\mathrm{H}]^{+}$} & $168(28) / 210(20)$ \\
\hline Dihydroergosine & 8.3 & $550(190)$ & {$[\mathrm{M}+\mathrm{H}]^{+}$} & $270(28) / 253(28)$ \\
\hline Dihydroergotamine & 8.5 & $584(190)$ & {$[\mathrm{M}+\mathrm{H}]^{+}$} & $270(32) / 254(32)$ \\
\hline Dihydrolysergol & 6.0 & $257(160)$ & {$[\mathrm{M}+\mathrm{H}]^{+}$} & $167(44) / 154(40)$ \\
\hline Elymoclavine & 5.9 & $255(135)$ & {$[\mathrm{M}+\mathrm{H}]^{+}$} & $181(28) / 180(44)$ \\
\hline Elymoclavine-fructoside & 5.5 & $417(165)$ & {$[\mathrm{M}+\mathrm{H}]^{+}$} & $255(16) / 237(20)$ \\
\hline Enniatin A & 15.5 & $699(170)$ & {$\left[\mathrm{M}+\mathrm{NH}_{4}\right]^{+}$} & $210(32) / 228(32)$ \\
\hline Enniatin A1 & 15.3 & $685(150)$ & {$\left[\mathrm{M}+\mathrm{NH}_{4}\right]^{+}$} & $210(32) / 228(32)$ \\
\hline Enniatin B & 14.7 & $657(160)$ & {$\left[\mathrm{M}+\mathrm{NH}_{4}\right]^{+}$} & $196(32) / 214(32)$ \\
\hline Enniatin B1 & 15.0 & $672(170)$ & {$\left[\mathrm{M}+\mathrm{NH}_{4}\right]^{+}$} & $196(32) / 100(60)$ \\
\hline Enniatin B2 & 14.1 & $643(145)$ & {$\left[\mathrm{M}+\mathrm{NH}_{4}\right]^{+}$} & $196(28) / 214(28)$ \\
\hline Enniatin B3 & 13.8 & $629(145)$ & {$\left[\mathrm{M}+\mathrm{NH}_{4}\right]^{+}$} & $196(28) / 214(28)$ \\
\hline Enniatin B4 & 14.7 & $671.5(170)$ & {$\left[\mathrm{M}+\mathrm{NH}_{4}\right]^{+}$} & $196(32) / 100(60)$ \\
\hline Enniatin K1 & 14.4 & $643(145)$ & {$\left[\mathrm{M}+\mathrm{NH}_{4}\right]^{+}$} & $196(28) / 214(28)$ \\
\hline Ergine + Erginine ${ }^{\mathrm{b}}$ & 5.5 & $268(125)$ & {$[\mathrm{M}+\mathrm{H}]^{+}$} & $223(16) / 208(24)$ \\
\hline Ergocornine & 8.6 & $562(165)$ & {$[\mathrm{M}+\mathrm{H}]^{+}$} & $208(48) / 223(36)$ \\
\hline Ergocorninine & 10.0 & $562(150)$ & {$[\mathrm{M}+\mathrm{H}]^{+}$} & $277(24) / 223(36)$ \\
\hline Ergocristine & 9.3 & $610(140)$ & {$[\mathrm{M}+\mathrm{H}]^{+}$} & $592.5(12) / 223(36)$ \\
\hline Ergocristinine & 11.0 & $610(140)$ & {$[\mathrm{M}+\mathrm{H}]^{+}$} & $592.5(12) / 223(36)$ \\
\hline Ergocryptine & 9.3 & $576(155)$ & {$[\mathrm{M}+\mathrm{H}]^{+}$} & $208(52) / 223(36)$ \\
\hline Ergocryptinine & 10.7 & $576(155)$ & {$[\mathrm{M}+\mathrm{H}]^{+}$} & $558.6(12) / 223(36)$ \\
\hline Ergometrine & 5.9 & $326(145)$ & {$[\mathrm{M}+\mathrm{H}]^{+}$} & $223(20) / 208(28)$ \\
\hline Ergometrinine & 6.5 & $326(145)$ & {$[\mathrm{M}+\mathrm{H}]^{+}$} & $223(20) / 208(28)$ \\
\hline Ergosine & 8.2 & $548(165)$ & {$[\mathrm{M}+\mathrm{H}]^{+}$} & $223(32) / 208(44)$ \\
\hline Ergosinine & 9.5 & $548(165)$ & {$[\mathrm{M}+\mathrm{H}]^{+}$} & $530.5(12) / 223(32)$ \\
\hline Ergotamine + Ergotaminine ${ }^{\mathrm{b}}$ & 8.5 & $582(155)$ & {$[\mathrm{M}+\mathrm{H}]^{+}$} & $223(32) / 208(48)$ \\
\hline Festuclavine & 7.0 & $241(160)$ & {$[\mathrm{M}+\mathrm{H}]^{+}$} & $154(36) / 168(28)$ \\
\hline Fulvic acid & 7.6 & $309(115)$ & {$[\mathrm{M}+\mathrm{H}]^{+}$} & $231(20) / 161(40)$ \\
\hline Fumagillin & 13.0 & $459(140)$ & {$[\mathrm{M}+\mathrm{H}]^{+}$} & $177(8) / 131(24)$ \\
\hline Fumigaclavin A & 6.6 & $299(170)$ & {$[\mathrm{M}+\mathrm{H}]^{+}$} & $167(48) / 154(44)$ \\
\hline Fumitremorgin $\mathrm{C}$ & 10.7 & $380(145)$ & {$[\mathrm{M}+\mathrm{H}]^{+}$} & $226(16) / 212(32)$ \\
\hline Fumonisin $\mathrm{B}_{1}$ & 10.3 & $722.5(210)$ & {$[\mathrm{M}+\mathrm{H}]^{+}$} & $352.3(36) / 334.4(44)$ \\
\hline Fumonisin $\mathrm{B}_{2}$ & 12.1 & $706.3(220)$ & {$[\mathrm{M}+\mathrm{H}]^{+}$} & $336.3(40) / 318.5(40)$ \\
\hline Fumonisin $\mathrm{B}_{3}$ & 11.3 & $706.5(220)$ & {$[\mathrm{M}+\mathrm{H}]^{+}$} & $336(40) / 318.5(40)$ \\
\hline Fusaproliferin & 14.6 & $445(105)$ & {$[\mathrm{M}+\mathrm{H}]^{+}$} & $385(4) / 367$ (4) \\
\hline Fusarielin A & 15.1 & $385(130)$ & {$\left[\mathrm{M}-\mathrm{H}_{2} \mathrm{O}+\mathrm{H}\right]^{+}$} & $109(16) / 253(8)$ \\
\hline Geodin & 11.4 & $399(125)$ & {$[\mathrm{M}+\mathrm{H}]^{+}$} & $340(20) / 355(4)$ \\
\hline Gibberellic acid & 7.0 & $364(105)$ & {$\left[\mathrm{M}+\mathrm{NH}_{4}\right]^{+}$} & $329(4) / 221(24)$ \\
\hline Gliotoxin & 8.8 & $327(95)$ & {$[\mathrm{M}+\mathrm{H}]^{+}$} & $263(4) / 245(16)$ \\
\hline Griseofulvin & 9.8 & $353(140)$ & {$[\mathrm{M}+\mathrm{H}]^{+}$} & $165(16) / 215(16)$ \\
\hline
\end{tabular}


Table 2 (continued)

\begin{tabular}{|c|c|c|c|c|}
\hline Analyte name & Retention time (min) & $\begin{array}{l}m / z \text { precursor ion } \\
\text { (framentor voltage) }\end{array}$ & Ion species & $\begin{array}{l}m / z \text { product ion (collision } \\
\text { energy }(\mathrm{V}) \text { ) }\end{array}$ \\
\hline $\mathrm{HC}$ toxin & 7.5 & $437(150)$ & {$[\mathrm{M}+\mathrm{H}]^{+}$} & $169(28) / 240(16)$ \\
\hline HT-2 toxin & 10.0 & $442.2(100)$ & {$\left[\mathrm{M}+\mathrm{NH}_{4}\right]^{+}$} & $263.1(8) / 215(4)$ \\
\hline Hydrolysed fumonisin $\mathrm{B}_{1}$ & 9.7 & $406(150)$ & {$[\mathrm{M}+\mathrm{H}]^{+}$} & $388(12) / 370(16)$ \\
\hline 16-Keto-aspergillimide & 13.1 & $374(185)$ & {$[\mathrm{M}+\mathrm{H}]^{+}$} & $313(36) / 315(28)$ \\
\hline Kojic acid & 2.5 & $143(120)$ & {$[\mathrm{M}+\mathrm{H}]^{+}$} & $69(16) / 97(12)$ \\
\hline Lysergol & 6.0 & $255(130)$ & {$[\mathrm{M}+\mathrm{H}]^{+}$} & $240(20) / 197(20)$ \\
\hline Malformin C & 12.6 & $530(205)$ & {$[\mathrm{M}+\mathrm{H}]^{+}$} & $417(12) / 372(16)$ \\
\hline Marcfortine A & 9.0 & $478(170)$ & {$[\mathrm{M}+\mathrm{H}]^{+}$} & $450(16) / 419(32)$ \\
\hline Meleagrin & 8.8 & $434(140)$ & {$[\mathrm{M}+\mathrm{H}]^{+}$} & $403(12) / 334(20)$ \\
\hline 3-O-Methylsterigmatocystin & 11.0 & $339(160)$ & {$[\mathrm{M}+\mathrm{H}]^{+}$} & $306(28) / 324(20)$ \\
\hline 3-O-Methylviridicatin & 11.3 & $252(155)$ & {$[\mathrm{M}+\mathrm{H}]^{+}$} & $236(28) / 190(48)$ \\
\hline Mevastatin & 13.9 & $408(95)$ & {$\left[\mathrm{M}+\mathrm{NH}_{4}\right]^{+}$} & $185(12) / 271(8)$ \\
\hline Mevinolin & 14.3 & $422(110)$ & {$\left[\mathrm{M}+\mathrm{NH}_{4}\right]^{+}$} & $199(4) / 173(24)$ \\
\hline 15-Monoacetoxyscirpenol & 7.9 & $342(95)$ & {$\left[\mathrm{M}+\mathrm{NH}_{4}\right]^{+}$} & $265(0) / 307(4)$ \\
\hline Mycophenolic acid & 10.7 & $338(75)$ & {$\left[\mathrm{M}+\mathrm{NH}_{4}\right]^{+}$} & $207(28) / 303(8)$ \\
\hline Neosolaniol & 6.6 & $400(110)$ & {$\left[\mathrm{M}+\mathrm{NH}_{4}\right]^{+}$} & $185(16) / 215(12)$ \\
\hline Neoxaline & 8.4 & $436(130)$ & {$[\mathrm{M}+\mathrm{H}]^{+}$} & $405(8) / 263(36)$ \\
\hline Ochratoxin A & 11.5 & $404(115)$ & {$[\mathrm{M}+\mathrm{H}]^{+}$} & $239(20) / 102(70)$ \\
\hline Ochratoxin B & 10.3 & $370(115)$ & {$[\mathrm{M}+\mathrm{H}]^{+}$} & $205(16) / 103(64)$ \\
\hline Ophiobolin A & 13.2 & $401(110)$ & {$[\mathrm{M}+\mathrm{H}]^{+}$} & $365(4) / 267(8)$ \\
\hline Ophiobolin B & 13.8 & $403(105)$ & {$[\mathrm{M}+\mathrm{H}]^{+}$} & $367(4) / 349(12)$ \\
\hline Oxaspirodione & 7.9 & $251(110)$ & {$[\mathrm{M}+\mathrm{H}]^{+}$} & $133(16) / 161(4)$ \\
\hline Paraherquamide A & 8.4 & $494(160)$ & {$[\mathrm{M}+\mathrm{H}]^{+}$} & $419(32) / 176(44)$ \\
\hline Paspalic acid & 5.5 & $269(140)$ & {$[\mathrm{M}+\mathrm{H}]^{+}$} & $182(28) / 167(44)$ \\
\hline Paxilline & 14.1 & $436(120)$ & {$[\mathrm{M}+\mathrm{H}]^{+}$} & $182(32) / 167(76)$ \\
\hline Penicillic acid & 6.6 & $171(70)$ & {$[\mathrm{M}+\mathrm{H}]^{+}$} & $125(8) / 97(12)$ \\
\hline Penicillin G & 6.8 & $335(95)$ & {$[\mathrm{M}+\mathrm{H}]^{+}$} & $160(4) / 176(8)$ \\
\hline Penitrem A & 13.9 & $634(165)$ & {$[\mathrm{M}+\mathrm{H}]^{+}$} & $558(16) / 616(4)$ \\
\hline Pentoxyfylline & 7.3 & $279(130)$ & {$[\mathrm{M}+\mathrm{H}]^{+}$} & $181(16) / 99(16)$ \\
\hline Pestalotin & 8.5 & $215(90)$ & {$[\mathrm{M}+\mathrm{H}]^{+}$} & $85(12) / 153(8)$ \\
\hline Phomopsin A & 7.3 & $789(205)$ & {$[\mathrm{M}+\mathrm{H}]^{+}$} & $226(40) / 323(24)$ \\
\hline Phomopsin B & 7.0 & $755(165)$ & {$[\mathrm{M}+\mathrm{H}]^{+}$} & $192(40) / 289(24)$ \\
\hline Pyripyropene A & 11.6 & $584(260)$ & {$[\mathrm{M}+\mathrm{H}]^{+}$} & $148(56) / 202(36)$ \\
\hline Roquefortine $\mathrm{C}$ & 10.2 & $390(145)$ & {$[\mathrm{M}+\mathrm{H}]^{+}$} & $193(24) / 322(16)$ \\
\hline Roridin A & 11.2 & $550(120)$ & {$\left[\mathrm{M}+\mathrm{NH}_{4}\right]^{+}$} & $249(12) / 231(20)$ \\
\hline Rugulosin & 12.4 & $543(145)$ & {$[\mathrm{M}+\mathrm{H}]^{+}$} & $273(20) / 255(36)$ \\
\hline Secalonic acid D & 13.3 & $639(220)$ & {$[\mathrm{M}+\mathrm{H}]^{+}$} & $561.4(24) / 589.4(24)$ \\
\hline Setosusin & 10.5 & $532(160)$ & {$\left[\mathrm{M}+\mathrm{NH}_{4}\right]^{+}$} & $413(20) / 299(28)$ \\
\hline Sterigmatocystin & 12.3 & $325(160)$ & {$[\mathrm{M}+\mathrm{H}]^{+}$} & $281(40) / 130(24)$ \\
\hline Sulochrin & 8.3 & $333(95)$ & {$[\mathrm{M}+\mathrm{H}]^{+}$} & $209(4) / 136(48)$ \\
\hline T-2 tetraol & 4.8 & $316(95)$ & {$\left[\mathrm{M}+\mathrm{NH}_{4}\right]^{+}$} & $215(4) / 281(4)$ \\
\hline $\mathrm{T}-2$ toxin & 10.9 & $484.3(120)$ & {$\left[\mathrm{M}+\mathrm{NH}_{4}\right]^{+}$} & $185.1(16) / 215.2(12)$ \\
\hline $\mathrm{T}-2$ triol & 9.1 & $400(105)$ & {$\left[\mathrm{M}+\mathrm{NH}_{4}\right]^{+}$} & $215(4) / 281(0)$ \\
\hline Terphenyllin & 9.1 & $339(125)$ & {$[\mathrm{M}+\mathrm{H}]^{+}$} & $307(8) / 292(20)$ \\
\hline Territrem B & 11.7 & $527(205)$ & {$[\mathrm{M}+\mathrm{H}]^{+}$} & $291(28) / 491(20)$ \\
\hline Trichodermin & 11.1 & $293(100)$ & {$[\mathrm{M}+\mathrm{H}]^{+}$} & $109(16) / 143(12)$ \\
\hline Tryprostatin A & 10.2 & $382(115)$ & {$[\mathrm{M}+\mathrm{H}]^{+}$} & $326(8) / 228(12)$ \\
\hline Ustiloxin A & 5.5 & $674(220)$ & {$[\mathrm{M}+\mathrm{H}]^{+}$} & $187(32) / 209(36)$ \\
\hline
\end{tabular}


Table 2 (continued)

\begin{tabular}{|c|c|c|c|c|}
\hline Analyte name & Retention time (min) & $\begin{array}{l}m / z \text { precursor ion } \\
\text { (framentor voltage) }\end{array}$ & Ion species & $\begin{array}{l}m / z \text { product ion (collision } \\
\text { energy }(\mathrm{V}) \text { ) }\end{array}$ \\
\hline Ustiloxin B & 4.7 & $646.2(170)$ & {$[\mathrm{M}+\mathrm{H}]^{+}$} & $181(36) / 187(28)$ \\
\hline Ustiloxin D & 5.7 & $495(135)$ & {$[\mathrm{M}+\mathrm{H}]^{+}$} & $192(20) / 291(12)$ \\
\hline Verrucarin A & 10.9 & $520(125)$ & {$\left[\mathrm{M}+\mathrm{NH}_{4}\right]^{+}$} & $249(12) / 457(8)$ \\
\hline Verrucofortine & 11.9 & $410(150)$ & {$[\mathrm{M}+\mathrm{H}]^{+}$} & $130(32) / 300(16)$ \\
\hline Verruculogen & 12.8 & $512(125)$ & {$[\mathrm{M}+\mathrm{H}]^{+}$} & $352(16) / 494(0)$ \\
\hline Viomellein & 12.5 & $561(215)$ & {$[\mathrm{M}+\mathrm{H}]^{+}$} & $530(28) / 511(32)$ \\
\hline Viridicatin & 11.2 & $238(155)$ & {$[\mathrm{M}+\mathrm{H}]^{+}$} & $165(36) / 192(24)$ \\
\hline Wortmannin & 8.8 & $429(95)$ & {$[\mathrm{M}+\mathrm{H}]^{+}$} & $355(4) / 295(20)$ \\
\hline
\end{tabular}

${ }^{a}$ In-source fragment obtained from the cleavage of the corresponding peptide bond

${ }^{\mathrm{b}}$ Due to co-elution and the same DMRM transitions, these analytes cannot be distinguished

were calculated and non-compliant values according to Commission Decision 2002/657/EC [29] were flagged automatically. Compared to a previously described method for the determination of 186 analytes including 164 fungal metabolites in dust [30], we describe a method for the determination of a total number of 191 fungal metabolites which include all relevant mycotoxins. Bacterial metabolites, which can occur in house dust, were eliminated from the former method as those metabolites are not relevant for food.

\section{Method performance parameters}

Method performance parameters were obtained by spiking blank samples before extraction on one medium level in triplicate and the raw extract of a blank sample after extraction on seven levels without replicates. It has to be pointed out that only $0.5 \mathrm{~g}$ of highly homogenised blank nuts was used for validation purposes to minimise the amount of spiked toxins. The results are presented in Tables 3, 4, 5 and 6 for almonds, hazelnuts, peanuts and pistachios, respectively. Spiking was performed with the whole multianalyte mix containing all metabolites, but data evaluation was restricted to the most important analytes, e.g. detected in the naturally contaminated nut samples, regulated mycotoxins or mycotoxins frequently found in other commodities. In total, method performance parameters for 65 analytes are shown and are discussed in the following. For about half of the analytes, apparent recoveries between 80 and $120 \%$ were achieved. This number was expected since a multi-target method covering a huge number of chemically diverse analytes always has to be a compromise. The chosen extraction solvent, as well as chromatographic conditions (regarding separation from matrix), was a compromise to include all analytes into the method. For example, in the case of ergot alkaloids, epimerisation is favoured under the used acidic conditions and, hence, only a rough estimate of a sum concentration is possible [31]. For the determination of specific analytes at a very high level of sensitivity, more dedicated methods (validated for the respective matrix) are recommended. These methods often also allow sample concentration as cleanup procedures can be employed. For the accurate determination of regulated mycotoxins, the use of stable isotope-labelled internal standards is also an excellent option. For instance, deuterated $\mathrm{AFB}_{2}$ and $\mathrm{AFG}_{2}$ had been used for the quantification of aflatoxins in almonds [32] or $11 \mathrm{U}-\left[{ }^{13} \mathrm{C}\right]$-labelled mycotoxins were applied in the analysis of maize [33].

In our case, the obtained standard deviation of $R_{\mathrm{A}}$ was below $10 \%$ for the vast majority of the analyte-matrix combinations. In only $6 \%$ of the cases were values above $15 \%$ determined, e.g. for enniatin A (13-23\%) and macrosporin (6-25\%). Significant deviations from $100 \%$ apparent recovery were either caused by severe matrix effects or insufficient extraction. Fifty-seven per cent of the analyte-matrix combinations showed SSE values between 80 and $120 \%$. Suppression of the analyte signal to more than the half compared to the neat standard was observed for early-eluting analytes 3-nitropropionic acid, moniliformin, kojic acid, as well as for the B-trichothecenes (nivalenol, DON and 3-acetyldeoxynivalenol) in all four matrices. Significant signal enhancement of more than $120 \%$ was observed for up to 17 analytes (almonds). The highest values were observed for physcion (246-295\%) and equisetin (231-285\%). Interestingly, for $\beta$-zearalenol-14-glucoside, a signal suppression to 54 and $79 \%$ for peanuts and almonds, respectively, were determined, whereas no matrix effect was monitored for pistachios (97\%) and a significant signal enhancement of $127 \%$ was observed in hazelnuts. This emphasises the importance of proper validation and indicates that samples belonging to the same food category can still show quite different matrix effects. The used solvent (acetonitrile/water/acetic acid (79:20:1, v/v/v) was well suited for the extraction of almost all analysed mycotoxins from the four different commodities. Ninety-four per cent of 
Table 3 Method performance characteristics determined for almonds

\begin{tabular}{lllll}
\hline Analyte & $\begin{array}{lll}\mathrm{LLOQ} \\
\left(\mu \mathrm{gkg}^{-1}\right)\end{array}$ & $\begin{array}{l}R_{\mathrm{A}}(\%) \pm \\
\mathrm{SD}^{\mathrm{a}}\end{array}$ & $\mathrm{SSE}(\%)^{\mathrm{b}}$ & $R_{\mathrm{E}}(\%)^{\mathrm{c}}$ \\
\hline
\end{tabular}

3-Acetyldeoxynivalenol

Aflatoxin $\mathrm{B}_{1}$

Aflatoxin $\mathrm{B}_{2}$

Aflatoxin $\mathrm{G}_{1}$

Aflatoxin $\mathrm{G}_{2}$

Aflatoxin $\mathrm{M}_{1}$

Alamethicin F30

alpha-Zearalenol

alpha-Zearalenol-14-

glucoside

Alternariol

Alternariolmethylether

Altertoxin I

Apidicin

Atpenin A5

Beauvericin

beta-Zearalenol

beta-Zearalenol-14-

glucoside

Chaetocin

Chlamydosporol

Curvularin

Cycloechinulin

Cyclopiazonic acid

Cyclosporin $\mathrm{A}+\mathrm{H}$

Cytochalasin $\mathrm{J}$

Deoxynivalenol

4,15-Diacetoxyscirpenol

Emodin

Enniatin A

Enniatin A1

Enniatin B

Enniatin B1

Enniatin B2

Enniatin B3

Enniatin B4

Enniatin K1

Equisetin

Fumonisin $\mathrm{B}_{1}$

Fumonisin $\mathrm{B}_{2}$

$\mathrm{HC}$ toxin

HT-2 toxin

Kojic acid

Macrosporin

Meleagrin

3-O-

Methylsterigmatocystin

Moniliformin

Mycophenolic acid

$48 \quad 51 \pm 22 \quad 45 \quad 112$

3.0

10

10

8.2

7.9

190

16

110

3.0

0.8

13

0.7

1.3

0.2

15

99

110

53

31

1.0

60

29

25

470

16

3.4

2.4

2.0

0.1

0.2

0.7

0.05

0.1

0.3

17

160

180

130

46

230

2.7

21

2.0

5.1

24
$51 \pm 22$

$101 \pm 6 \quad 87$

$81 \pm 10 \quad 81$

$78 \pm 1 \quad 78$

$85 \pm 1 \quad 67$

$73 \pm 3 \quad 70$

$33 \pm 5 \quad 88$

$125 \pm 7 \quad 137$

$98 \pm 10 \quad 126$

$115 \pm 5 \quad 126$

$117 \pm 7 \quad 108$

$95 \pm 4 \quad 104$

$98 \pm 4 \quad 117$

$124 \pm 8 \quad 123$

$86 \pm 8 \quad 85$

$143 \pm 2 \quad 145$

$68 \pm 5 \quad 79$

$29 \pm 1 \quad 126$

$101 \pm 4 \quad 78$

$195 \pm 12 \quad 187$

$124 \pm 13 \quad 166$

$66 \pm 4 \quad 70$

$96 \pm 6 \quad 94$

$22 \pm 4 \quad 19$

$104 \pm 8 \quad 92$

$120 \pm 9 \quad 150$

$106 \pm 23 \quad 81$

$76 \pm 18 \quad 77$

$100 \pm 11 \quad 107$

$84 \pm 8 \quad 83$

$108 \pm 8 \quad 96$

$88 \pm 8 \quad 99$

$95 \pm 5 \quad 97$

$222 \pm 11 \quad 285$

$41 \pm 4 \quad 100$

$38 \pm 7 \quad 96$

$35 \pm 9 \quad 58$

$113 \pm 8 \quad 95$

$34 \pm 2 \quad 38$

$165 \pm 20 \quad 146$

$104 \pm 9 \quad 95$

$99 \pm 5 \quad 97$

$15 \pm 1 \quad 17$

$101 \pm 8 \quad 94$
$107 \pm 10 \quad 105$

$107 \pm 11 \quad 99$
Table 3 (continued)

\begin{tabular}{lllll}
\hline Analyte & $\begin{array}{l}\text { LLOQ } \\
\left(\mu \mathrm{kgg}^{-1}\right)\end{array}$ & $\begin{array}{l}R_{\mathrm{A}}(\%) \pm \\
\mathrm{SD}^{\mathrm{a}}\end{array}$ & $\mathrm{SSE}(\%)^{\mathrm{b}}$ & $R_{\mathrm{E}}(\%)^{\mathrm{c}}$ \\
\hline Neoxaline & 3.0 & $93 \pm 7$ & 103 & 90 \\
Nidulin & 0.5 & $73 \pm 3$ & 99 & 74 \\
3-Nitropropionic acid & 5.8 & $45 \pm 4$ & 46 & 98 \\
Nivalenol & 44 & $14 \pm 3$ & 12 & 123 \\
Nornidulin & 1.2 & $111 \pm 8$ & 125 & 88 \\
Ochratoxin A & 15 & $90 \pm 8$ & 89 & 101 \\
Ochratoxin B & 9.9 & $84 \pm 5$ & 94 & 89 \\
Penigequinolone A & 0.4 & $142 \pm 14$ & 150 & 95 \\
Pestalotin & 6.0 & $112 \pm 11$ & 98 & 114 \\
Physcion & 260 & $155 \pm 13$ & 293 & 53 \\
Pseurotin A & 25 & $159 \pm 12$ & 173 & 92 \\
Roquefortine C & 5.5 & $136 \pm 7$ & 97 & 141 \\
Secalonic acid D & 41 & $77 \pm 5$ & 99 & 78 \\
Sterigmatocystin & 2.8 & $106 \pm 7$ & 108 & 98 \\
T-2 toxin & 5.2 & $105 \pm 9$ & 101 & 104 \\
Tentoxin & 1.2 & $111 \pm 4$ & 120 & 92 \\
Viridicatin & 7.5 & $104 \pm 7$ & 100 & 104 \\
Zearalenone & 5.3 & $127 \pm 6$ & 122 & 104 \\
Zearalenone-14-sulphate & 5.2 & $62 \pm 6$ & 86 & 72 \\
\hline
\end{tabular}

$L L O Q$ lower limit of quantification

${ }^{\text {a }}$ Apparent recovery and standard deviation thereof (triplicate analysis on medium level)

${ }^{\mathrm{b}}$ Mean value for the signal suppression or enhancement calculated from up to seven concentration levels without replicates

${ }^{\mathrm{c}}$ Calculated extraction recovery $\left(R_{\mathrm{A}} / \mathrm{SSE} \times 100\right)$

the analyte-matrix combinations showed extraction recoveries higher than $50 \%$. Lower $R_{\mathrm{E}}$ values were for example observed for fumonisins (29 and $42 \%$ ), which were even lower than reported before for wheat (43-53\%) and maize (57-67\%) using the same solvent mixture for extraction [4]. In cases of severe matrix suppression, e.g. SSE of $8 \%$ for DON in pistachios, also unreliable $R_{\mathrm{E}}$ values were calculated (e.g. $225 \%$ for DON in pistachios).

The linear range covered two orders of magnitude in most cases. For atpenin A5, even a linear range of three orders of magnitude was observed in all four matrices. The LLOQs varied between sub-microgram per kilogram levels, e.g. for apicidin, beauvericin and enniatins, and up to $500 \mu \mathrm{gkg}^{-1}$ for $\mathrm{HC}$ toxin in hazelnuts. It should be pointed out here that in some cases (e.g. altertoxin I, atpenin A5 or neoxaline), even the lowest spiking level showed an S/N ratio above 10. All shown LLOQs will undoubtedly be higher compared to calculations based on $\mathrm{S} / \mathrm{N}$ ratios equalling 10 . We decided on purpose for this very conservative calculation of the LLOQs to minimize the influence of dayto-day performance differences, which are often encountered in mass spectrometry due to staining of the ion path. 
Table 4 Method performance characteristics determined for hazelnuts

\begin{tabular}{|c|c|c|c|c|}
\hline Analyte & $\begin{array}{l}\text { LLOQ } \\
\left(\mu \mathrm{gkg}^{-1}\right)\end{array}$ & $\begin{array}{l}R_{\mathrm{A}}(\%) \pm \\
\mathrm{SD}^{\mathrm{a}}\end{array}$ & $\operatorname{SSE}(\%)^{\mathrm{b}}$ & $R_{\mathrm{E}}(\%)$ \\
\hline 3-Acetyldeoxynivalenol & 54 & $55 \pm 6$ & 55 & 100 \\
\hline Aflatoxin $\mathrm{B}_{1}$ & 10 & $97 \pm 13$ & 98 & 99 \\
\hline Aflatoxin $\mathrm{B}_{2}$ & 11 & $87 \pm 5$ & 90 & 97 \\
\hline Aflatoxin $\mathrm{G}_{1}$ & 4.4 & $81 \pm 6$ & 103 & 79 \\
\hline Aflatoxin $\mathrm{G}_{2}$ & 11 & $83 \pm 8$ & 89 & 93 \\
\hline Aflatoxin $\mathrm{M}_{1}$ & 9.8 & $68 \pm 7$ & 81 & 84 \\
\hline Alamethicin F30 & 210 & $31 \pm 7$ & 90 & 35 \\
\hline alpha-Zearalenol & 18 & $99 \pm 12$ & 122 & 81 \\
\hline $\begin{array}{l}\text { alpha-Zearalenol-14- } \\
\text { glucoside }\end{array}$ & 490 & $71 \pm 9$ & 121 & 58 \\
\hline Alternariol & 3.0 & $95 \pm 7$ & 107 & 89 \\
\hline Alternariolmethylether & 2.9 & $95 \pm 7$ & 101 & 94 \\
\hline Altertoxin I & 14 & $90 \pm 5$ & 106 & 85 \\
\hline Apidicin & 0.8 & $77 \pm 9$ & 113 & 69 \\
\hline Atpenin A5 & 1.6 & $104 \pm 10$ & 127 & 82 \\
\hline Beauvericin & 0.6 & $77 \pm 3$ & 89 & 87 \\
\hline beta-Zearalenol & 16 & $106 \pm 8$ & 118 & 89 \\
\hline $\begin{array}{l}\text { beta-Zearalenol-14- } \\
\text { glucoside }\end{array}$ & 170 & $64 \pm 6$ & 127 & 51 \\
\hline Chaetocin & 180 & $23 \pm 1$ & 158 & 14 \\
\hline Chlamydosporol & 69 & $107 \pm 3$ & 107 & 100 \\
\hline Curvularin & 33 & $97 \pm 3$ & 105 & 93 \\
\hline Cycloechinulin & 3.6 & $132 \pm 13$ & 138 & 96 \\
\hline Cyclopiazonic acid & 56 & $121 \pm 23$ & 149 & 81 \\
\hline Cyclosporin $\mathrm{A}+\mathrm{H}$ & 36 & $57 \pm 7$ & 73 & 78 \\
\hline Cytochalasin J & 34 & $77 \pm 4$ & 101 & 76 \\
\hline Deoxynivalenol & 46 & $40 \pm 8$ & 34 & 118 \\
\hline 4,15-Diacetoxyscirpenol & 19 & $97 \pm 6$ & 101 & 96 \\
\hline Emodin & 3.9 & $99 \pm 9$ & 143 & 70 \\
\hline Enniatin A & 3.0 & $73 \pm 20$ & 72 & 102 \\
\hline Enniatin A1 & 0.5 & $83 \pm 6$ & 73 & 114 \\
\hline Enniatin B & 0.1 & $99 \pm 8$ & 104 & 95 \\
\hline Enniatin B1 & 0.2 & $78 \pm 7$ & 82 & 95 \\
\hline Enniatin B2 & 1.0 & $82 \pm 7$ & 105 & 79 \\
\hline Enniatin B3 & 0.1 & $92 \pm 6$ & 100 & 92 \\
\hline Enniatin B4 & 0.1 & $75 \pm 1$ & 104 & 72 \\
\hline Enniatin K1 & 0.5 & $74 \pm 2$ & 107 & 69 \\
\hline Equisetin & 17 & $188 \pm 15$ & 242 & 78 \\
\hline Fumonisin $\mathrm{B}_{1}$ & 190 & $37 \pm 5$ & 105 & 35 \\
\hline Fumonisin $\mathrm{B}_{2}$ & 240 & $31 \pm 3$ & 109 & 29 \\
\hline $\mathrm{HC}$ toxin & 500 & $31 \pm 7$ & 61 & 51 \\
\hline HT-2 toxin & 59 & $94 \pm 7$ & 100 & 93 \\
\hline Kojic acid & 280 & $34 \pm 3$ & 47 & 73 \\
\hline Macrosporin & 3.2 & $146 \pm 25$ & 150 & 98 \\
\hline Meleagrin & 19 & $95 \pm 4$ & 79 & 120 \\
\hline $\begin{array}{l}\text { 3-O- } \\
\text { Methylsterigmatocystin }\end{array}$ & 2.4 & $89 \pm 3$ & 103 & 86 \\
\hline Moniliformin & 5.3 & $14 \pm 1$ & 17 & 84 \\
\hline Mycophenolic acid & 27 & $93 \pm 6$ & 100 & 92 \\
\hline
\end{tabular}

Table 4 (continued)

\begin{tabular}{lllll}
\hline Analyte & $\begin{array}{l}\left.\mathrm{LLOQ}^{-1}\right) \\
\left(\mu \mathrm{gkg}^{-1}\right)\end{array}$ & $\begin{array}{l}R_{\mathrm{A}}(\%) \pm \\
\mathrm{SD}^{\mathrm{a}}\end{array}$ & $\mathrm{SSE}(\%)^{\mathrm{b}}$ & $R_{\mathrm{E}}(\%)^{\mathrm{c}}$ \\
\hline Neoxaline & 3.5 & $97 \pm 10$ & 128 & 76 \\
Nidulin & 0.6 & $61 \pm 4$ & 103 & 60 \\
3-Nitropropionic acid & 7.1 & $36 \pm 2$ & 45 & 79 \\
Nivalenol & 86 & $16 \pm 1$ & 26 & 63 \\
Nornidulin & 0.5 & $93 \pm 12$ & 143 & 65 \\
Ochratoxin A & 18 & $73 \pm 7$ & 92 & 80 \\
Ochratoxin B & 14 & $67 \pm 3$ & 108 & 62 \\
Penigequinolone A & 0.5 & $116 \pm 20$ & 157 & 74 \\
Pestalotin & 6.2 & $109 \pm 3$ & 98 & 111 \\
Physcion & 270 & $129 \pm 6$ & 252 & 51 \\
Pseurotin A & 3.0 & $111 \pm 19$ & 143 & 78 \\
Roquefortine C & 6.0 & $119 \pm 9$ & 93 & 128 \\
Secalonic acid D & 49 & $69 \pm 10$ & 106 & 65 \\
Sterigmatocystin & 3.1 & $87 \pm 3$ & 99 & 88 \\
T-2 toxin & 5.6 & $90 \pm 7$ & 93 & 96 \\
Tentoxin & 1.2 & $93 \pm 12$ & 107 & 87 \\
Viridicatin & 8.3 & $96 \pm 5$ & 103 & 94 \\
Zearalenone & 2.2 & $91 \pm 7$ & 111 & 82 \\
Zearalenone-14-sulphate & 7.8 & $47 \pm 5$ & 100 & 47 \\
\hline
\end{tabular}

$L L O Q$ lower limit of quantification

${ }^{\text {a }}$ Apparent recovery and standard deviation thereof (triplicate analysis on medium level)

${ }^{b}$ Mean value for the signal suppression or enhancement calculated from up to seven concentration levels without replicates

${ }^{\mathrm{c}}$ Calculated extraction recovery $\left(R_{\mathrm{A}} / \mathrm{SSE} \times 100\right)$

Still, the obtained recoveries and LLOQs were in a similar range as those reported before for peanuts [28], with the exception of DON due to signal suppression in our case. However, the gained standard deviations were lower in our proposed method, which can be partly explained by the combination of three different blank materials by Warth and co-workers, whereas we used one blank material and spiked it in triplicate. In comparison to [5], both for peanuts and pistachios, our method proved to be more repeatable for almost all analytes. The limits of detection were significantly lower for aflatoxins, OTA and fumonisins in the method proposed by Spanjer et al. [5], whilst they were lower for, e.g. zearalenone and T-2 toxin in our method. Compared to our method, lesser matrix effects were obtained by Ediage et al. [27] after extensive cleanup for 25 mycotoxins.

The gained LLOQs for aflatoxins are higher than the maximum levels set in Commission Regulation (EC) No. $1881 / 2006$ and its amendments [3], rendering this method unsuited for regulatory purposes. Still, the presented method can be an appropriate supplement to already existing singleanalyte or analyte-group detection methods which usually have been developed specifically for the respective target 
Table 5 Method performance characteristics determined for peanuts

\begin{tabular}{lllll}
\hline Analyte & $\begin{array}{l}\mathrm{LLOQ} \\
\left(\mu \mathrm{kg}^{-1}\right)\end{array}$ & $\mathrm{R}_{\mathrm{A}}(\%) \pm$ & $\mathrm{SSE}(\%)^{\mathrm{b}}$ & $R_{\mathrm{E}}(\%)^{\mathrm{c}}$ \\
\hline
\end{tabular}

3-Acetyldeoxynivalenol

Aflatoxin $\mathrm{B}_{1}$

Aflatoxin $\mathrm{B}_{2}$

Aflatoxin $\mathrm{G}_{1}$

Aflatoxin $\mathrm{G}_{2}$

Aflatoxin $\mathrm{M}_{1}$

Alamethicin F30

alpha-Zearalenol

alpha-Zearalenol-14-

glucoside

Alternariol

Alternariolmethylether

Altertoxin I

Apidicin

Atpenin A5

Beauvericin

beta-Zearalenol

beta-Zearalenol-14-

glucoside

Chaetocin

Chlamydosporol

Curvularin

Cycloechinulin

Cyclopiazonic acid

Cyclosporin $\mathrm{A}+\mathrm{H}$

Cytochalasin J

Deoxynivalenol

4,15-Diacetoxyscirpenol

Emodin

Enniatin A

Enniatin A1

Enniatin B

Enniatin B1

Enniatin B2

Enniatin B3

Enniatin B4

Enniatin K1

Equisetin

Fumonisin $\mathrm{B}_{1}$

Fumonisin $\mathrm{B}_{2}$

$\mathrm{HC}$ toxin

HT-2 toxin

Kojic acid

Macrosporin

Meleagrin

3-O-

Methylsterigmatocystin

Moniliformin

Mycophenolic acid

190

10

10

12

9.4

8.7

110

14

97

8.8

8.8

2.7

14

0.6

1.2

0.4

15

99

120

16

29

3.1

61

28

26

26
150

18

11

2.8

1.4

0.05

0.1

2.4

0.04

0.2

0.3

13

210

180

130

50

280

2.6

16

1.9

6.3

26
$17 \pm 1$

$84 \pm 9$

$84 \pm 4$

17

17
85

96

$67 \pm 5$

82

99

$73 \pm 4$

75

$65 \pm 3$

66

69

91

$107 \pm 2 \quad 108$

$104 \pm 2 \quad 117$

$101 \pm 3 \quad 98$

$84 \pm 1 \quad 84$

$79 \pm 2 \quad 87$

$97 \pm 3 \quad 107$

$132 \pm 3 \quad 126$

$94 \pm 8 \quad 82$

$115 \pm 2 \quad 119$

$46 \pm 2 \quad 54$

$24 \pm 1 \quad 114$

$125 \pm 9 \quad 97$

$107 \pm 4 \quad 100$

$148 \pm 5 \quad 135$

$84 \pm 2 \quad 114$

$64 \pm 8 \quad 64$

$92 \pm 4 \quad 92$

$29 \pm 1 \quad 24$

$92 \pm 4 \quad 91$

$122 \pm 6 \quad 151$

$86 \pm 17 \quad 79$

$101 \pm 37 \quad 72$

$117 \pm 16 \quad 81$

$102 \pm 8 \quad 89$

$95 \pm 11 \quad 87$

$100 \pm 3 \quad 79$

$97 \pm 4 \quad 92$

$98 \pm 8 \quad 88$

$267 \pm 13 \quad 276$

$33 \pm 8 \quad 102$

$40 \pm 3 \quad 105$

$18 \pm 1 \quad 30$

$99 \pm 8 \quad 90$

$27 \pm 1$

$166 \pm 6 \quad 137$

$107 \pm 2 \quad 73$

$88 \pm 2 \quad 82$

$16 \pm 1 \quad 23$

$84 \pm 3 \quad 87$
Table 5 (continued)

\begin{tabular}{lllll}
\hline Analyte & $\begin{array}{l}\mathrm{LLOQ} \\
\left(\mu \mathrm{gkg}^{-1}\right)\end{array}$ & $\begin{array}{l}\mathrm{R}_{\mathrm{A}}(\%) \pm \\
\mathrm{SD}^{\mathrm{a}}\end{array}$ & $\mathrm{SSE}(\%)^{\mathrm{b}}$ & $R_{\mathrm{E}}(\%)^{\mathrm{c}}$ \\
\hline Neoxaline & 2.8 & $113 \pm 4$ & 119 & 95 \\
Nidulin & 0.5 & $75 \pm 1$ & 98 & 76 \\
3-Nitropropionic acid & 6.9 & $51 \pm 1$ & 62 & 82 \\
Nivalenol & 69 & $19 \pm 1$ & 25 & 78 \\
Nornidulin & 1.2 & $118 \pm 1$ & 137 & 86 \\
Ochratoxin A & 17 & $78 \pm 3$ & 89 & 88 \\
Ochratoxin B & 37 & $77 \pm 5$ & 96 & 80 \\
Penigequinolone A & 1.0 & $146 \pm 5$ & 139 & 105 \\
Pestalotin & 6.2 & $101 \pm 5$ & 91 & 111 \\
Physcion & 260 & $161 \pm 12$ & 295 & 54 \\
Pseurotin A & 23 & $152 \pm 9$ & 150 & 102 \\
Roquefortine C & 5.2 & $94 \pm 5$ & 63 & 149 \\
Secalonic acid D & 38 & $105 \pm 5$ & 125 & 84 \\
Sterigmatocystin & 2.4 & $97 \pm 3$ & 84 & 115 \\
T-2 toxin & 5.1 & $94 \pm 7$ & 88 & 107 \\
Tentoxin & 1.1 & $108 \pm 5$ & 115 & 93 \\
Viridicatin & 7.8 & $95 \pm 2$ & 96 & 100 \\
Zearalenone & 5.7 & $109 \pm 1$ & 112 & 97 \\
Zearalenone-14-sulphate & 5.2 & $65 \pm 2$ & 94 & 69 \\
\hline
\end{tabular}

$L L O Q$ lower limit of quantification

${ }^{a}$ Apparent recovery and standard deviation thereof (triplicate analysis on medium level)

${ }^{\mathrm{b}}$ Mean value for the signal suppression or enhancement calculated from up to seven concentration levels without replicates

${ }^{\mathrm{c}}$ Calculated extraction recovery $\left(R_{\mathrm{A}} / \mathrm{SSE} \times 100\right)$

analytes in certain matrices. Alternatives to gain the needed sensitivity are cleanup and enrichment of aflatoxins (e.g. by immunoaffinity columns), a method parameter set which is optimised on those compounds, or the use of a more sensitive mass spectrometer [33]. Finally, albeit the method was validated for nuts, it is also applicable for the screening of mycotoxins in several other food and feed.

Naturally contaminated nut samples

Information about the occurrence of other mycotoxins than aflatoxins and OTA in nuts is very limited in the literature. We were able to show the applicability of the method through the analysis of 53 different nut ( 8 almonds, 22 hazelnuts, 15 peanuts and 8 pistachios) samples. Further microbial investigations about the source and time point of contamination are warranted and the scope of future experiments. Figure 1 provides an overview of the detected mycotoxins in the analysed samples and presents the percentage of contaminated nut samples compared to all analysed samples of the respective analyte. In Table 7, more detailed information including the average concentration of contaminated samples and the 
Table 6 Method performance characteristics determined for pistachios

Analyte

$$
\text { LLOQ } \quad R_{\mathrm{A}}(\%) \pm \quad \operatorname{SSE}(\%)^{\mathrm{b}} \quad R_{\mathrm{E}}(\%)^{\mathrm{c}}
$$
$\left(\mu \mathrm{gkg}^{-1}\right) \quad \mathrm{SD}^{\mathrm{a}}$

3-Acetyldeoxynivalenol

Aflatoxin $\mathrm{B}_{1}$

Aflatoxin $\mathrm{B}_{2}$

Aflatoxin $\mathrm{G}_{1}$

Aflatoxin $\mathrm{G}_{2}$

Aflatoxin $\mathrm{M}_{1}$

Alamethicin F30

alpha-Zearalenol

alpha-Zearalenol-14-

glucoside

Alternariol

Alternariolmethylether

Altertoxin I

Apidicin

Atpenin A5

Beauvericin

beta-Zearalenol

beta-Zearalenol-14-

glucoside

Chaetocin

Chlamydosporol

Curvularin

Cycloechinulin

Cyclopiazonic acid

Cyclosporin $\mathrm{A}+\mathrm{H}$

Cytochalasin $\mathrm{J}$

Deoxynivalenol

4,15-Diacetoxyscirpenol

Emodin

Enniatin A

Enniatin A1

Enniatin B

Enniatin B1

Enniatin B2

Enniatin B3

Enniatin B4

Enniatin K1

Equisetin

Fumonisin $\mathrm{B}_{1}$

Fumonisin $\mathrm{B}_{2}$

$\mathrm{HC}$ toxin ${ }^{\mathrm{d}}$

HT-2 toxin

Kojic acid

Macrosporin

Meleagrin

3-O-

Methylsterigmatocystin

Moniliformin

Mycophenolic acid

\section{5}

10

11

10

11

8.9

243

17

100

9.6

0.9

14

0.7

1.4

0.6

15

120

260

200

100

1.1

58

36

28

241

5.4

3.5

2.5

2.3

0.1

0.2

0.8

0.1

0.1

0.3

15

200

220

\section{5}

230

3.1

19

7.4

17

26

\section{$37 \pm 5$}

$85 \pm 6$

$42 \pm 3$

31

$69 \pm 6$

83

43

$62 \pm 11$

69

$60 \pm 6$

64

$28 \pm 3$

$67 \pm 5 \quad 80$

$97 \pm 1 \quad 102$

$107 \pm 2 \quad 107$

$91 \pm 2 \quad 101$

$82 \pm 2 \quad 110$

$121 \pm 3 \quad 131$

$72 \pm 10 \quad 78$

$119 \pm 7 \quad 126$

$72 \pm 1 \quad 97$

$33 \pm 1 \quad 112$

$83 \pm 3 \quad 82$

$100 \pm 8 \quad 100$

$129 \pm 7 \quad 134$

$131 \pm 6 \quad 168$

$59 \pm 1 \quad 75$

$85 \pm 8 \quad 91$

$18 \pm 4 \quad 8$

$92 \pm 4 \quad 91$

$114 \pm 2 \quad 147$

$95 \pm 13 \quad 76$

$65 \pm 4 \quad 77$

$105 \pm 37 \quad 93$

$83 \pm 11 \quad 79$

$80 \pm 2 \quad 88$

$90 \pm 1 \quad 97$

$83 \pm 2 \quad 99$

$89 \pm 18 \quad 87$

$193 \pm 7 \quad 231$

$37 \pm 1 \quad 107$

$34 \pm 3 \quad 110$

$96 \pm 7 \quad 96$

$28 \pm 1 \quad 32$

$172 \pm 17 \quad 171$

$80 \pm 2 \quad 67$

$91 \pm 3 \quad 98$

$16 \pm 1 \quad 19$

$92 \pm 4 \quad 95$
Table 6 (continued)

\begin{tabular}{lllll}
\hline Analyte & $\begin{array}{l}\left.\mathrm{LLOQ}^{-1}\right) \\
\left(\mu \mathrm{gkg}^{-1}\right)\end{array}$ & $\begin{array}{l}R_{\mathrm{A}}(\%) \pm \\
\mathrm{SD}^{\mathrm{a}}\end{array}$ & $\mathrm{SSE}(\%)^{\mathrm{b}}$ & $R_{\mathrm{E}}(\%)^{\mathrm{c}}$ \\
\hline Neoxaline $^{\mathrm{d}}$ & 1.6 & $62 \pm 2$ & 98 & 63 \\
Nidulin & 19 & $39 \pm 1$ & 44 & 87 \\
3-Nitropropionic acid & 95 & $6 \pm 2$ & 3 & 189 \\
Nivalenol & 1.4 & $105 \pm 3$ & 141 & 74 \\
Nornidulin & 18 & $79 \pm 3$ & 94 & 84 \\
Ochratoxin A & 10 & $75 \pm 1$ & 86 & 87 \\
Ochratoxin B & 1.3 & $132 \pm 4$ & 162 & 82 \\
Penigequinolone A & 6.9 & $93 \pm 3$ & 93 & 99 \\
Pestalotin & 76 & $150 \pm 6$ & 246 & 61 \\
Physcion & 28 & $156 \pm 13$ & 185 & 84 \\
Pseurotin A & 5.6 & $111 \pm 1$ & 81 & 137 \\
Roquefortine C & 48 & $73 \pm 5$ & 110 & 67 \\
Secalonic acid D & 2.7 & $93 \pm 5$ & 94 & 99 \\
Sterigmatocystin & 5.5 & $95 \pm 5$ & 97 & 98 \\
T-2 toxin & 1.1 & $101 \pm 2$ & 108 & 94 \\
Tentoxin & 8.0 & $94 \pm 0.2$ & 97 & 97 \\
Viridicatin & 6.1 & $104 \pm 2$ & 117 & 89 \\
Zearalenone & 5.6 & $67 \pm 5$ & 97 & 69 \\
Zearalenone-14-sulphate & & & & \\
\hline
\end{tabular}

$L L O Q$ lower limit of quantification

${ }^{a}$ Apparent recovery and standard deviation thereof (triplicate analysis on medium level)

${ }^{\mathrm{b}}$ Mean value for the signal suppression or enhancement calculated from up to seven concentration levels without replicates

${ }^{\mathrm{c}}$ Calculated extraction recovery $\left(R_{\mathrm{A}} / \mathrm{SSE} \times 100\right)$

${ }^{\mathrm{d}}$ Due to interferences, it was not possible to evaluate HC toxin and neoxaline in pistachios

maximum observed level is given. In total, the presence of 40 analytes could be confirmed in different kinds of nuts. Most analytes were determined in hazelnuts (36), followed by peanuts (30), almonds (13) and pistachios (5). The reported mycotoxins are produced by a wide range of food spoilage and contaminating fungal species, including Aspergillus spp. (e.g. aflatoxins, sterigmatocystin); Fusarium spp. (e.g. ennitatins, equisetin); Penicillium spp. (e.g. mycophenolic acid, roquefortine C); and Alternaria spp. (e.g. alternariol, macrosporin). The most prevalent mycotoxin was beauvericin, which was identified in 42 samples, followed by enniatin B (33), macrosporin (30) and 3-nitropropionic acid (29). Beauvericin is a depsipeptide produced by various Fusarium spp.; concentrations up to $31 \mu \mathrm{gkg}^{-1}$ in hazelnuts and $12 \mu \mathrm{g} \mathrm{kg}^{-1}$ in peanuts were observed. These values are in very good accordance to the occurrence of beauvericin in peanuts $\left(0.1-24.0 \mu \mathrm{gkg}^{-1}, 73 \%\right.$ positive samples) reported before [28]. The contamination range was higher than reported by [26] in peanut cake $\left(0.05-3.36 \mu \mathrm{gkg}^{-1}\right)$. Furthermore, several Alternaria mycotoxins were found in nuts, with 
Fig. 1 Overview of the detected mycotoxins in the analysed samples

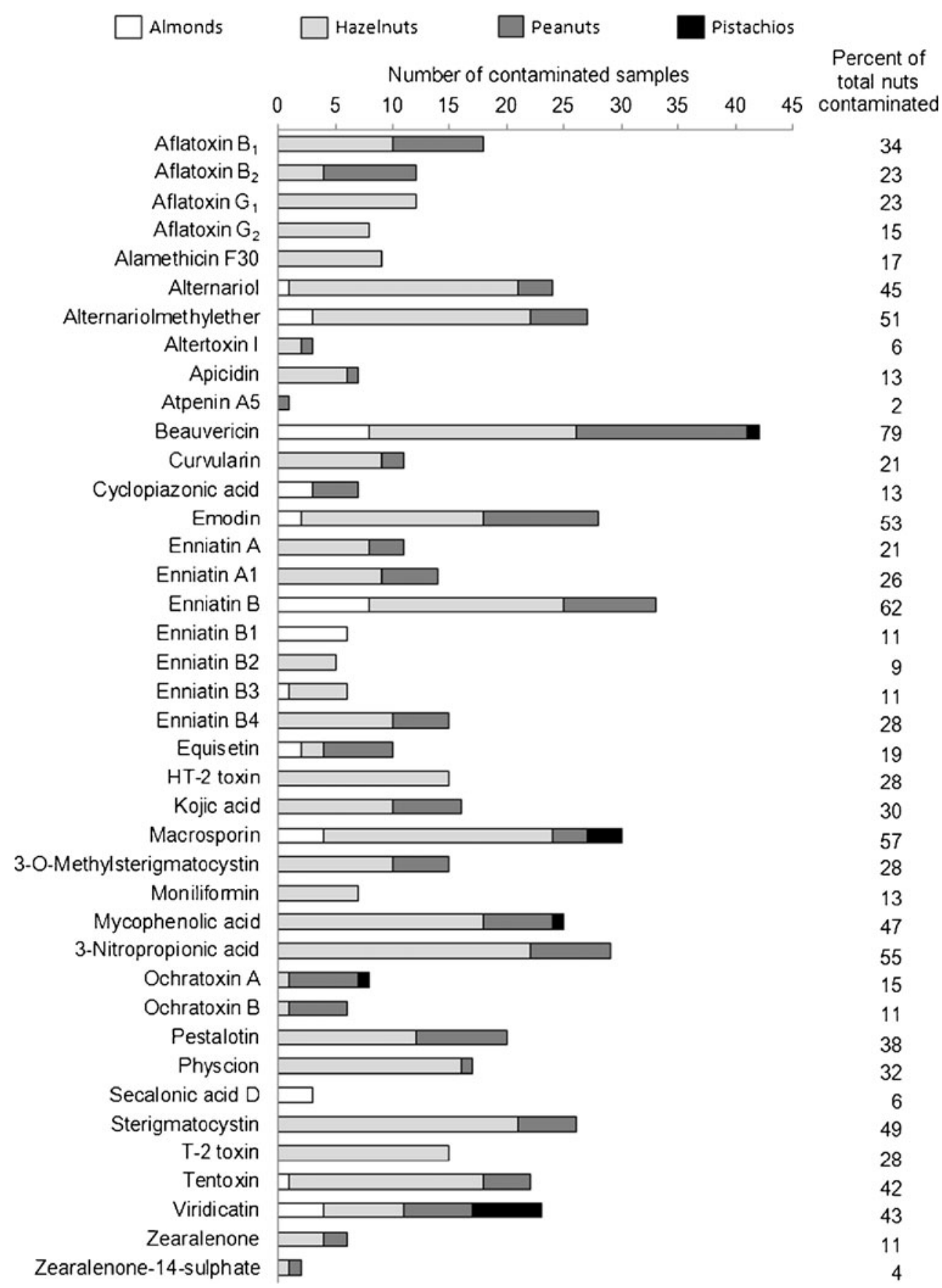

alternariolmethylether (27 samples), alternariol (24 samples) and tentoxin (22 samples) being the most prevalent. Also tenuazonic acid was detected in 21 samples, with apparently high concentration, although this analyte was not part of the validated compounds. Emodin was found in 28 out of 53 samples, which is in agreement with the occurrence of this Aspergillus mycotoxin reported previously in inoculated peanuts [34]. In one hazelnut sample, 26 analytes were determined, and eight other hazelnut samples were contaminated with 20 or more mycotoxins. In peanuts, almonds and pistachios, up to 17,13 and 5 analytes, respectively, were detected in one sample.

Almond and especially pistachio samples were contaminated to a lesser extent than peanut and hazelnut samples. However, it has to be kept in mind that the sample size was also smaller for those matrices. In the case of pistachios, only five analytes could be determined and only one sample was contaminated above the LLOQ (11 $\mathrm{\mu gkg}^{-1}$ macrosporin). Concerning almonds, 15 analytes were determined, among others Alternaria toxins and enniatins. The highest 
Table 7 Mycotoxin contamination of analysed nut samples

\begin{tabular}{|c|c|c|c|c|c|c|c|c|}
\hline \multirow[t]{2}{*}{ Analyte } & \multicolumn{2}{|c|}{ Almonds $(n=8)$} & \multicolumn{2}{|c|}{ Hazelnuts $(n=22)$} & \multicolumn{2}{|c|}{ Peanuts $(n=15)$} & \multicolumn{2}{|c|}{ Pistachios $(n=8)$} \\
\hline & $\begin{array}{l}\text { Avg. }{ }^{\mathrm{a}} \\
\left(\mu \mathrm{gkg}{ }^{-1}\right)\end{array}$ & $\begin{array}{l}\operatorname{Max}^{\mathrm{b}} \\
\left(\mu \mathrm{gkg}^{-1}\right)\end{array}$ & $\begin{array}{l}\text { Avg. }{ }^{\mathrm{a}} \\
\left(\mu \mathrm{gkg}^{-1}\right)\end{array}$ & $\begin{array}{l}\text { Max. }^{\mathrm{b}} \\
\left(\mu \mathrm{gkg}^{-1}\right)\end{array}$ & $\begin{array}{l}\text { Avg. }{ }^{\mathrm{a}} \\
\left(\mu \mathrm{gkg}^{-1}\right)\end{array}$ & $\begin{array}{l}\text { Max. }^{\mathrm{b}} \\
\left(\mu \mathrm{gkg}^{-1}\right)\end{array}$ & $\begin{array}{l}\text { Avg. }{ }^{\mathrm{a}} \\
\left(\mu \mathrm{gkg}{ }^{-1}\right)\end{array}$ & $\begin{array}{l}\text { Max. }^{\mathrm{b}} \\
\left(\mu \mathrm{gkg}{ }^{-1}\right)\end{array}$ \\
\hline Aflatoxin $\mathrm{B}_{1}$ & - & n.d. & $7.4(10)$ & 15 & $68(8)$ & 230 & - & n.d. \\
\hline Aflatoxin $\mathrm{B}_{2}$ & - & n.d. & $5.5(4)$ & $<$ LLOQ & $11(8)$ & 22 & - & n.d. \\
\hline Aflatoxin $\mathrm{G}_{1}$ & - & n.d. & $16(12)$ & 28 & - & n.d. & - & n.d. \\
\hline Aflatoxin $\mathrm{G}_{2}$ & - & n.d. & $5.5(8)$ & $<$ LLOQ & - & n.d. & - & n.d. \\
\hline Alamethicin F30 & - & n.d. & $110(9)$ & $<$ LLOQ & - & n.d. & - & n.d. \\
\hline Alternariol & $1.5(1)$ & $<$ LLOQ & $78(20)$ & 650 & $4.4(3)$ & $<$ LLOQ & - & n.d. \\
\hline Alternariolmethylether & $0.56(3)$ & 0.84 & $59(19)$ & 220 & $1.4(5)$ & $<$ LLOQ & - & n.d. \\
\hline Altertoxin I & - & n.d. & $7.0(2)$ & $<$ LLOQ & - & $<$ LLOQ & - & n.d. \\
\hline Apicidin & - & n.d. & $3.4(6)$ & 14 & - & $<$ LLOQ & - & n.d. \\
\hline Atpenin A5 & - & n.d. & - & n.d. & - & $<$ LLOQ & - & n.d. \\
\hline Beauvericin & $0.10(8)$ & $<$ LLOQ & $2.4(18)$ & 31 & $1.6(15)$ & 12 & $0.30(1)$ & $<$ LLOQ \\
\hline Curvularin & - & n.d. & $19(9)$ & 42 & $19(2)$ & 24 & - & n.d. \\
\hline Cyclopiazonic acid & $64(3)$ & 130 & - & n.d. & $140(4)$ & 310 & - & n.d. \\
\hline Emodin & $1.7(2)$ & $<$ LLOQ & $5.5(16)$ & 23 & $5.5(10)$ & $<$ LLOQ & - & n.d. \\
\hline Enniatin A & - & n.d. & $28(8)$ & 170 & $1.4(3)$ & $<$ LLOQ & - & n.d. \\
\hline Enniatin A1 & - & n.d. & $140(9)$ & 1,100 & $0.70(5)$ & $<$ LLOQ & - & n.d. \\
\hline Enniatin B & $0.05(8)$ & $<$ LLOQ & $37(17)$ & 540 & $0.23(8)$ & 0.45 & - & n.d. \\
\hline Enniatin B1 & $0.12(6)$ & 0.21 & - & n.d. & - & n.d. & - & n.d. \\
\hline Enniatin B2 & - & n.d. & $3.0(5)$ & 8.9 & - & n.d. & - & n.d. \\
\hline Enniatin B3 & $0.05(1)$ & 0.05 & $0.06(5)$ & 0.11 & - & n.d. & - & n.d. \\
\hline Enniatin B4 & - & n.d. & $22(10)$ & 190 & $0.1(5)$ & $<$ LLOQ & - & n.d. \\
\hline Equisetin & $8.5(2)$ & $<$ LLOQ & $110(2)$ & 200 & $14(6)$ & 41 & - & n.d. \\
\hline HT-2 toxin & - & n.d. & $39(15)$ & 130 & - & n.d. & - & n.d. \\
\hline Kojic acid & - & n.d. & $1,100(10)$ & 1,400 & $8,900(6)$ & 40,000 & - & n.d. \\
\hline Macrosporin & $2.1(4)$ & 4.4 & $280(20)$ & 2,200 & $9.4(3)$ & 26 & $4.6(3)$ & 11 \\
\hline $\begin{array}{l}\text { 3-O-Methyl- } \\
\text { sterigmatocystin }\end{array}$ & - & n.d. & $1.7(10)$ & 3.9 & $1.6(5)$ & 2.7 & - & n.d. \\
\hline Moniliformin & - & n.d. & $5.8(7)$ & 9.2 & - & n.d. & - & n.d. \\
\hline Mycophenolic acid & - & n.d. & $700(18)$ & 6,100 & $21(6)$ & 60 & $13(1)$ & $<$ LLOQ \\
\hline 3-Nitropropionic acid & - & n.d. & $440(22)$ & 980 & $82(7)$ & 350 & - & n.d. \\
\hline Ochratoxin A & - & n.d. & $220(1)$ & 220 & $67(6)$ & 260 & $9.0(1)$ & $<$ LLOQ \\
\hline Ochratoxin B & - & n.d. & $6.9(1)$ & 6.9 & $31(5)$ & 82 & - & n.d. \\
\hline Pestalotin & - & n.d. & $3.1(12)$ & $<$ LLOQ & $5.4(8)$ & 21 & - & n.d. \\
\hline Physcion & - & n.d. & $700(16)$ & 3,300 & - & $<$ LLOQ & - & n.d. \\
\hline Secalonic acid D & $31(3)$ & 51 & - & n.d. & - & n.d. & - & n.d. \\
\hline Sterigmatocystin & - & n.d. & $2.3(21)$ & 5.5 & $1.2(5)$ & $<$ LLOQ & - & n.d. \\
\hline $\mathrm{T}-2$ toxin & - & n.d. & $32(15)$ & 40 & - & n.d. & - & n.d. \\
\hline Tentoxin & $0.60(1)$ & $<$ LLOQ & $5.4(17)$ & 21 & $4.7(4)$ & 11 & - & n.d. \\
\hline Viridicatin & $3.8(4)$ & $<$ LLOQ & $5.7(7)$ & 15 & $3.9(6)$ & $<$ LLOQ & $4.0(6)$ & $<$ LLOQ \\
\hline Zearalenone & - & n.d. & $7.6(4)$ & 21 & $2.9(2)$ & $<$ LLOQ & - & n.d. \\
\hline Zearalenone-14-sulphate & - & n.d. & $3.9(1)$ & $<$ LLOQ & $2.6(1)$ & $<$ LLOQ & - & n.d. \\
\hline
\end{tabular}

$n$ total number of samples analysed in this category, n.d. not detected, $\angle L L O Q$ below the lower limit of quantification

${ }^{a}$ Average of samples with detectable amounts of the specific analyte; in parentheses, the number of samples used for the calculation is given (in the case of detectable levels which are below the LLOQ, half of the LLOQ of the respective matrix was used for calculation)

${ }^{\mathrm{b}}$ Highest determined concentration 
contamination was observed for cyclopiazonic acid (up to $130 \mu \mathrm{gkg}^{-1}$ ) and secalonic acid D (up to $51 \mu \mathrm{gkg}^{-1}$ ).

Some hazelnut samples showed significant contamination, including aflatoxins (up to $15 \mu \mathrm{g} \mathrm{kg}^{-1} \mathrm{AFB}_{1}$ and $28 \mu \mathrm{gkg}^{-1}$ $\mathrm{AFG}_{1}$ ) and Alternaria mycotoxins (up to $650 \mu \mathrm{g} \mathrm{kg}^{-1}$ alternariol and $220 \mu \mathrm{g} \mathrm{kg}^{-1}$ alternariolmethylether). The determined $\mathrm{AFB}_{1}$ concentrations were above the maximum level set in the European Union $\left(2.0 / 5.0 / 8.0 / 8.0 \mu \mathrm{gkg}^{-1}\right.$ $\mathrm{AFB}_{1}$ in peanuts, hazelnuts, almonds and pistachios, respectively) [3] for eight hazelnut and eight peanut samples. 3-Nitropropionic acid (up to $980 \mu \mathrm{g} \mathrm{kg}^{-1}$ ) was determined in all hazelnut samples. Sterigmatocystin (up to $5.5 \mu \mathrm{g} \mathrm{kg}^{-1}$ ), the most toxic $\mathrm{AFB}_{1}$ precursor, was found in 21 out of the 22 analysed hazelnut samples. We were also able to identify T-2 and HT-2 toxins in nut samples for the first time ever. Whereas the occurrence of these toxins was never confirmed in nuts, several Fusarium spp. including the T-2- and HT-2-producing Fusarium oxysporum were detected on almonds and pistachios before [35]. Average values of the contaminated 15 samples were 39 and $32 \mathrm{\mu gkg}^{-1}$ for the HT-2 and T-2 toxins, respectively. In 17 hazelnut samples, enniatin B was detected reaching levels up to $540 \mu \mathrm{g} \mathrm{kg}^{-1}$. The highest concentration of all enniatins was about $1100 \mathrm{\mu g} \mathrm{kg}^{-1}$ in a single hazelnut sample, whilst in no other commodity were enniatins found above $0.5 \mathrm{\mu gkg}^{-1}$. With regard to mycophenolic acid, a potent immunosuppressive compound, one sample was contaminated with $6,100 \mu \mathrm{gkg}^{-1}$. In addition, macrosporin was found up to $2,200 \mu \mathrm{gkg}^{-1}$.

In peanuts, 32 analytes were detected. The highest contamination was observed for a sample containing $40 \mathrm{mgkg}^{-1}$ kojic acid; the most prevalent was beauvericin, which was detected in all samples with an average concentration of $1.6 \mu \mathrm{g} \mathrm{kg}^{-1}$ and a maximum of $12 \mu \mathrm{g} \mathrm{kg}^{-1}$. As already pointed out in $[28,36]$, we could confirm the presence of cyclopiazonic acid, a mycotoxin produced, e.g. by Aspergillus flavus, in four peanut samples up to a concentration of $310 \mu \mathrm{gkg}^{-1}$. This is a lower contamination rate as reported before $[28,36]$. Also, for 3-nitropropionic acid, previously determined concentrations [28] have been verified.

\section{Conclusion}

In conclusion, an UHPLC-MS/MS-based method for the determination of 191 mycotoxins and other fungal metabolites has been developed. Compared to other LC-MS/MS methods, UHPLC allowed better separation of the analytes from the matrix. Whilst most methods for the determination of mycotoxins focus on cereals or cereal-based foods, we developed a method for almonds, hazelnuts, peanuts and pistachios. An in-house validation for 65 analytes was performed, allowing the quantification of those analytes in the four commodities. For the other 126 analytes, the method still can provide semiquantitative information about the degree of contamination, and additional analytes might be validated afterwards, if needed. The overall repeatability of the proposed method is superior to currently published methods.

The method is based on a fast and easy sample preparation, including a single extraction step and subsequent injection of the diluted raw extract into the UHPLC-MS/MS system without any sample cleanup. Two chromatographic runs for each sample allow a throughput of about 25 samples per day (including standards). As with all multi-target methods, the major bottleneck regarding sample throughput is data evaluation, which is quite laborious and timeconsuming. Various software tools, in particular "compound at a glance" or flagging options, can greatly assist in this process, though.

Finally, the method has been applied to the analysis of 53 different nut samples. In total, 40 different analytes were detected, showing the importance of multimycotoxin methods. It seems that besides aflatoxins, the only mycotoxins regulated in nuts in the European Union, other toxins might also be relevant. The obtained mycotoxin pattern shows that a variety of fungal species, including Aspergillus spp., Penicillium spp., Fusarium spp. and Alternaria spp., might grow on nuts and are capable of producing a variety of toxins. The most prominent mycotoxins found in more than $50 \%$ of the samples were beauvericin, ennitatin B, macrosporin, 3nitropropionic acid, emodin and alternariolmethylether. Additionally, we could, for the first time, confirm the presence of HT-2 and T-2 toxin in hazelnuts. For many of the detected mycotoxins, possible toxic effects on humans are still not fully evaluated. Even more, possible additive or synergistic effects of co-occurring toxins are largely unknown. The major benefit of the developed method is its usage in the (semi-)quantitative screening for a large number of mycotoxins and other fungal metabolites in nuts and food in general.

Acknowledgments The authors thank the Federal Ministry of Economy, Family and Youth, the National Foundation for Research, Technology and Development, BIOMIN Holding GmbH and Nestec Ltd. for funding the Christian Doppler Laboratory for Mycotoxin Metabolism. Furthermore, we want to thank the European Commission for financial support within the Project MycoRed (KBBE-2007-22269-2). We also thank Agilent Technologies for providing the UHPLC-MS/MS instrument and Dr. Ahmet D. Duman from the Department of Food Engineering of the Faculty of Agriculture of the Kahramanmaras Sutcu Imam University (KSU) in Turkey for providing hazelnut and peanut samples. Additionally, we want to acknowledge all the research groups who provided us with standards (in alphabetical order): Erica Bloom, Anders Broberg, Hans Brückner, Miroslav Flieger, David Gilchrist, Marika Jestoi, Matthias Koch, Kristian Fog Nielsen, Herbert Oberacher, Danka Pericic, Michele Solfrizzo and Silvio Uhlig. 
Open Access This article is distributed under the terms of the Creative Commons Attribution License which permits any use, distribution, and reproduction in any medium, provided the original author(s) and the source are credited.

\section{References}

1. Zöllner P, Mayer-Helm B (2006) Trace mycotoxin analysis in complex biological and food matrices by liquid chromatographyatmospheric pressure ionisation mass spectrometry. J Chromatogr A $1136: 123-169$

2. Bennett JW, Klich M (2003) Mycotoxins. Clin Microbiol Rev $16: 497-516$

3. Commission Regulation (EC) No. 1881/2006 of 19 December 2006 setting maximum levels for certain contaminants in foodstuffs - consolidated version 01 Sept (2012). European Community, Brussels. http://eur-lex.europa.eu/LexUriServ/LexUriServ.do? uri=CONSLEG:2006R1881:20120901:EN:PDF. Accessed 31 December 2012

4. Sulyok M, Berthiller F, Krska R, Schuhmacher R (2006) Development and validation of a liquid chromatography/tandem mass spectrometric method for the determination of 39 mycotoxins in wheat and maize. Rapid Commun Mass Spectrom 20:2649-2659

5. Spanjer MC, Rensen PM, Scholten JM (2008) LC-MS/MS multimethod for mycotoxins after single extraction, with validation data for peanut, pistachio, wheat, maize, cornflakes, raisins and figs. Food Addit Contam 25:472-489

6. Ren Y, Zhang Y, Shao S, Cai Z, Feng L, Pan H, Wang Z (2007) Simultaneous determination of multi-component mycotoxin contaminants in foods and feeds by ultra-performance liquid chromatography tandem mass spectrometry. J Chromatogr A 1143:48-64

7. Sulyok M, Krska R, Schuhmacher R (2007) A liquid chromatography/tandem mass spectrometric multi-mycotoxin method for the quantification of 87 analytes and its application to semi-quantitative screening of moldy food samples. Anal Bioanal Chem 389:1505-1523

8. Zachariasova M, Lacina O, Malachova A, Kostelanska M, Poustka J, Godula M, Hajslova J (2010) Novel approaches in analysis of Fusarium mycotoxins in cereals employing ultra performance liquid chromatography coupled with high resolution mass spectrometry. Anal Chim Acta 662:51-61

9. Mol HGJ, Plaza-Bolaños P, Zomer P, de Rijk TC, Stolker AAM, Mulder PPJ (2008) Toward a generic extraction method for simultaneous determination of pesticides, mycotoxins, plant toxins, and veterinary drugs in feed and food matrixes. Anal Chem 80:9450 9459

10. Boonzaaijer G, van Osenbruggen WA, Kleinnijenhuis AJ, van Dongen WD (2008) An exploratory investigation of several mycotoxins and their natural occurrence in flavor ingredients and spices, using a multi-mycotoxin LC-MS/MS method. World Mycotoxin J 1:167-174

11. Lattanzio VMT, Solfrizzo M, Visconti A (2008) Determination of trichothecenes in cereals and cereal-based products by liquid chromatography-tandem mass spectrometry. Food Addit Contam Part A 25:320-330

12. Tanaka H, Takino M, Sugita-Konishi Y, Tanaka T (2006) Development of a liquid chromatography/time-of-flight mass spectrometric method for the simultaneous determination of trichothecenes, zearalenone and aflatoxins in foodstuffs. Rapid Commun Mass Spectrom 20:1422-1428

13. Berthiller F, Schuhmacher R, Buttinger G, Krska R (2005) Rapid simultaneous determination of major type A- and Btrichothecenes as well as zearalenone in maize by high performance liquid chromatography-tandem mass spectrometry. J Chromatogr A 1062:209-216

14. Lattanzio VMT, Solfrizzo M, Powers S, Visconti A (2007) Simultaneous determination of aflatoxins, ochratoxin A and Fusarium toxins in maize by liquid chromatography/tandem mass spectrometry after multitoxin immunoaffinity cleanup. Rapid Commun Mass Spectrom 21:3253-3261

15. Rasmussen RR, Storm IMLD, Rasmussen PH, Smedsgaard J, Nielsen KF (2010) Multi-mycotoxin analysis of maize silage by LC-MS/MS. Anal Bioanal Chem 397:765-776

16. Desmarchelier A, Oberson J-M, Tella P, Gremaud E, Seefelder W, Mottier P (2010) Development and comparison of two multiresidue methods for the analysis of 17 mycotoxins in cereals by liquid chromatography electrospray ionization tandem mass spectrometry. J Agric Food Chem 58:7510-7519

17. Alasalvar C, Shahidi F (2009) Tree nuts: composition, phytochemicals, and health effects. CRC, Boca Raton

18. Frisvad JC, Andersen B, Samson RA (2007) Association of moulds to foods. In: Dijksterhuis J, Samson RA (eds) Food mycology: a multifaceted approach to fungi and food. CRC, Boca Raton

19. Bayman P, Baker JL, Doster MA, Michailides TJ, Mahoney NE (2002) Ochratoxin production by the Aspergillus ochraceus group and Aspergillus alliaceus. Appl Environ Microbiol 68:2326-2329

20. Pitt JI, Hocking AD (1997) Fungi and food spoilage, 2nd edn. Blackie Academic and Professional, London

21. Shahidi Bonjar GH (2004) Incidence of aflatoxin producing fungi in early split pistachio nuts of Kerman, Iran. J Biol Sci 4:199-202

22. Rodrigues P, Venâncio A, Lima N (2012) Mycobiota and mycotoxins of almonds and chestnuts with special reference to aflatoxins. Food Res Int 48:76-90

23. Hayat A, Paniel N, Rhouati A, Marty J-L, Barthelmebs L (2012) Recent advances in ochratoxin A-producing fungi detection based on PCR methods and ochratoxin A analysis in food matrices. Food Cont 26:401-415

24. Shephard GS (2009) Aflatoxin analysis at the beginning of the twenty-first century. Anal Bioanal Chem 395:1215-1224

25. Sulyok M, Krska R, Schuhmacher R (2010) Application of an LCMS/MS based multi-mycotoxin method for the semi-quantitative determination of mycotoxins occurring in different types of food infected by moulds. Food Chem 119:408-416

26. Ezekiel CN, Sulyok M, Warth B, Odebode AC, Krska R (2012) Natural occurrence of mycotoxins in peanut cake from Nigeria. Food Cont 27:338-342

27. Ediage EN, Di Mavungu JD, Monbaliu S, Peteghem CV, De Saeger S (2011) A validated multianalyte LC-MS/MS method for the quantification of 25 mycotoxins in cassava flour, peanut cake and maize samples. J Agric Food Chem 59:5173-5180

28. Warth B, Parich A, Atehnkeng J, Bandyopadhyay R, Schuhmacher R, Sulyok M, Krska R (2012) Quantitation of mycotoxins in food and feed from Burkina Faso and Mozambique using a modern LC-MS/MS multitoxin method. J Agric Food Chem 60:9352-9363

29. Commission Decision No. 2002/657/EC of 14 August 2002 implementing Council Directive 96/23/EC concerning the performance of analytical methods and the interpretation of results - consolidated version 10 Jan 2004. http://eur-lex.europa.eu/LexUriServ/ LexUriServ.do?uri=CONSLEG:2002D0657:20040110:EN:PDF. Accessed 31 December 2012

30. Vishwanath V, Sulyok M, Labuda R, Bicker W, Krska R (2009) Simultaneous determination of 186 fungal and bacterial metabolites in indoor matrices by liquid chromatography/ tandem mass spectrometry. Anal Bioanal Chem 395:13551372

31. Lehner AF, Craig M, Fannin N, Bush L, Tobin T (2005) Electrospray $[+]$ tandem quadrupole mass spectrometry in the 
elucidation of ergot alkaloids chromatographed by HPLC: screening of grass or forage samples for novel toxic compounds. J Mass Spectrom 40:1484-1502

32. Cervino C, Asam S, Knopp D, Rychlik M, Niessner R (2008) Use of isotope-labeled aflatoxins for LC-MS/MS stable isotope dilution analysis of foods. J Agric Food Chem 56:1873-1879

33. Varga E, Glauner T, Köppen R, Mayer K, Sulyok M, Schuhmacher R, Krska R, Berthiller F (2012) Stable isotope dilution assay for the accurate determination of mycotoxins in maize by UHPLCMS/MS. Anal Bioanal Chem 402:2675-2686
34. Hasan HAH (1998) Studies on toxigenic fungi in roasted foodstuff (salted seed) and halotolerant activity of emodin-producing Aspergillus wentii. Folia Microbiol 43:383-391

35. Abdel-Gawad KM, Zohri AA (1993) Fungal flora and mycotoxins of six kinds of nut seeds for human consumption in Saudi Arabia. Mycopathologia 124:55-64

36. Zorzete $\mathrm{P}$, Reis TA, Felício JD, Baquião AC, Makimoto $\mathrm{P}$, Corrêa B (2011) Fungi, mycotoxins and phytoalexin in peanut varieties, during plant growth in the field. Food Chem 129:957-964 